University of Zurich

Department of Economics

Working Paper Series

ISSN 1664-7041 (print)

ISSN 1664-705X (online)

Working Paper No. 126

\title{
Limelight on Dark Markets: Theory and Experimental Evidence on Liquidity and Information
}

Aleksander Berentsen, Michael McBride and Guillaume Rocheteau

Revised version, April 2015 


\title{
Limelight on Dark Markets: Theory and Experimental Evidence on Liquidity and Information*
}

\author{
Aleksander Berentsen \\ University of Basel \\ Federal Reserve Bank of St.Louis
}

\author{
Michael McBride \\ University of California, Irvine \\ Experimental Social Science Laboratory
}

Guillaume Rocheteau

University of California, Irvine

April 24, 2015

\begin{abstract}
We investigate how informational frictions affect trading in decentralized markets in theory and in a laboratory setting. Subjects, matched pairwise at random, trade divisible commodities that have different private values for a divisible asset with a common value (interpreted as money). We compare a bargaining game with complete information with a bargaining game where agents can produce fraudulent assets at some cost and are privately informed about the quality of their assets. The threat of fraud strongly reduces the subjects' ability to exploit the gains from trade, it reduces significantly both the size of the trade and the acceptability of the asset, but only a small fraction of all assets are actually fraudulent.
\end{abstract}

JEL Classification: G12, G14, E42, D82, D83

Keywords: liquidity, money, information, experiments

\footnotetext{
*Berentsen (aleksander.berentsen@unibas.ch): Department of Economics, University of Basel, Switzerland and Federal Reserve Bank of St.Louis. McBride (mcbride@uci.edu): Department of Economics, University of California-Irvine, USA. Rocheteau (grochete@uci.edu): Department of Economics, University of California-Irvine, USA. For comments on earlier versions of this paper, we thank David Brownstone, Gabriele Camera, Christian Kleiber, Daniela Puzzello, participants of the Summer Workshop on Money, Banking, Payments and Finance at the Federal Reserve Bank in Chicago, the Vienna Macroeconomics Workshop, the annual meeting of the Swiss Society of Economics and Statistics, the Economic Science Association, the conference on Behavioral Aspects in Macroeconomics and Finance in Milan and seminar participants at the Banque de France, Chapman University, the University of California, Santa Barbara, the University of Cologne, the University of Melbourne, the University of Oxford, and the Einaudi Institute for Economics and Finance (EIEF) in Rome. This research benefited from the financial support of the Foundation Banque de France. McBride also acknowledges financial support from the Air Force Office of Scientific Research Award No. FA9550-10-1-0569 and the Army Research Office Award No. W911NF-11-1-0332. We thank the Experimental Social Science Laboratory at UC Irvine for use of its facilities. We also thank Michael Caldara, Matthew Hicks, Ryan Kendall, Garret Ridinger, Anne Carpenter, and Si-Yuan Kong for research assistance. The usual disclaimer applies.
} 
"Cognizability: By this name we may denote the capability of a substance for being easily recognized and distinguished from all other substances. As a medium of exchange, money has to be continually handed about, and it will occasion great trouble if every person receiving currency has to scrutinize, weigh, and test it. If it requires any skill to discriminate good money from bad, poor ignorant people are sure to be imposed upon. Hence the medium of exchange should have certain distinct marks which nobody can mistake." Jevons (1875, Chapter 5)

\section{Introduction}

As far back as Jevons (1875), at least, it has been commonly accepted that a key property of a monetary asset-broadly defined as an asset that serves as a means of payment or collateral-is its recognizability, the fact that an asset can be authenticated at little cost. Throughout monetary history assets that lacked recognizability have been subjected to counterfeiting and fraud, e.g., the clipping of coins in ancient Rome and medieval Europe, and the counterfeiting of banknotes during the first half of the $19^{\text {th }}$ century in the United States (Sargent and Velde, 2002; Mihm, 2007). More recently, problems arising from private information problems and fraud regarding collateral have played a crucial role in the unfolding of the 2007-08 financial crisis and the drying-up of liquidity in over-the-counter (OTC) markets, such as the market for bilateral repurchase agreements (repos). ${ }^{1}$ According to Duffie $(2012$, p.2) private information problems are prevalent in decentralized asset markets with search and bargaining:

"An OTC bargaining game can be complex because of private information (...). The counterparties may have different information regarding the common-value aspects of the asset (for example, the probability distribution of the asset's future cash flows), current market conditions, and their individual motives for trade."

The search-theoretic approach to monetary exchange (Shi, 1995; Trejos and Wright, 1995) and OTC trading (Duffie, Gârleanu, and Pedersen, 2005) provides theoretical foundations for decentralized marketsmarkets with pairwise meetings and bargaining - in which agents trade assets (e.g., money, real assets, financial securities) in the absence of private information. ${ }^{2}$ This literature has been recently extended to

\footnotetext{
${ }^{1}$ Prior to the 2008 crisis, asset-backed securities (ABSs) were used as collateral, and trillions of dollars were exchanged on the repo market without any extensive due diligence (Gorton and Metrick, 2010). When market participants realized that ABSs could be of dubious quality and the private information of asset holders became relevant, assets that had served as collateral were subject to prohibitive haircuts and liquidity in money markets dried up dramatically.

${ }^{2}$ Trejos and Wright (2014) provide a framework nesting the Duffie-Garleanu-Pedersen model of OTC trading and the ShiTrejos-Wright model of monetary exchange.
} 
introduce the possibility of counterfeiting and asset fraud and to analyze bargaining games under private information, e.g., Nosal and Wallace (2007), Li and Rocheteau (2011), Li, Rocheteau, and Weill (2012), $\mathrm{Hu}$ (2013) and Shao (2014). These models have proven useful to explain how the threat of fraud affects the liquidity of assets (e.g., their acceptability and pledgeability) as well as the coexistence of assets with different rates of return - a puzzle in monetary theory. Moreover, they provide a simple narrative for the recent financial crisis - a class of assets became vulnerable to fraudulent practices, which reduced aggregate liquidity and led to a flight to quality.

There are two concerns with this theoretical literature, both of which can be addressed by an experimental approach. First, models of bargaining in decentralized markets with informational frictions generate different sets of predictions depending on the equilibrium notion that is used. For example, Li, Rocheteau, and Weill (2012), under a reordering invariance refinement, and Shao (2014), under the notion of competitive search, find that the threat of fraud reduces the trade size in a match, but not the acceptability of offers. In contrast, under the intuitive criterion, Nosal and Wallace (2007) found that the threat of fraud leads to a complete shut-down of the market. The fact that equilibrium allocations are sensitive to the choice of the equilibrium concept generates a need to confront these theories with real observations. Unfortunately, decentralized markets are dark markets - a term coined by Duffie (2012) — for which relatively little information is made publicly available. This makes it difficult to confront these theories with data obtained from these markets. An experimental approach can fill this gap, thus allowing for a sharper test of equilibrium predictions.

A second concern is the stark prediction of these models according to which counterfeiting is not an equilibrium phenomenon - it is an out-of-equilibrium threat - unless fraud is costless. This result holds for both signaling games where asset holders with private information make offers (e.g., Nosal and Wallace, 2007; Li, Rocheteau, and Weill, 2012; Hu, 2013) or screening games where the buyers of the asset are the ones to make offers (e.g., Shao, 2014). ${ }^{3}$ This prediction of the theory has important policy implications as it suggests that measures to make media of exchange recognizable should be implemented irrespective of realized fraud. In actual economies, however, fraud and counterfeiting do happen. For instance, in 2005, out

\footnotetext{
${ }^{3}$ There are exceptions. Li and Rocheteau (2011) obtain counterfeiting in equilibrium by introducing some price posting in the Nosal-Wallace model. However, as shown by Shao (2014), this result is not robust if search is directed. Li, Rocheteau, and Weill (2012) obtain counterfeiting in equilibrium in one extension of their model where the cost of fraud is random but offers cannot be made contingent on that cost.
} 
of the $\$ 760$ billion of U.S. banknotes in circulation $\$ 61$ million of counterfeit currency was passed on to the public. ${ }^{4}$ The experimental approach can help us understand if counterfeiting corresponds to a behavioral response to the possibility of fraud that is not captured by our model or whether it is an out-of-equilibrium phenomenon that vanishes as subjects learn about the environment. It can also reveal if the residual amount of counterfeiting taking place in actual economies can serve as an indicator of the degree of recognizability of a medium of exchange.

The environment we implement in a laboratory setting is an extension of the decentralized market with random matching and bargaining under private information of Li, Rocheteau, and Weill (2012), LRW thereafter. In contrast to LRW we impose linear payoffs, which makes the environment easier to apprehend for the subjects but also generates new insights for the effects of the threat of fraud on asset liquidity. Under a natural timing assumption, our modified bargaining game has a multiplicity of Perfect Bayesian Nash equilibria. To address this issue, we apply the reordering invariance refinement of In and Wright (2011) for signaling games with hidden actions. This refinement generates a limited set of equilibria that are payoff equivalent and all feature the same no-counterfeiting property. Relative to LRW, our game has the novel implication that it can generate equilibria with both limited pledgeability (i.e., agents only trade a fraction of their asset holdings) and partial acceptability of assets (i.e., a fraction of offers get rejected) - the two key dimensions of asset (il)liquidity. ${ }^{5}$ Moreover, equilibria feature a trade-off between trade sizes and acceptability—larger offers take longer to be accepted. Finally, we extend the theory to take into account fairness considerations that typically play a role in experiments, and we do so in a way that is consistent with a bargaining solution commonly used in monetary theory, namely, the proportional bargaining solution. ${ }^{6}$

\footnotetext{
${ }^{4}$ See "The use and counterfeiting of United States currency abroad," Part 3, page 47. Also, according to the estimate by Judson and Porter (2003) counterfeit U.S. currency that has been passed into circulation is about one note in ten thousands of currency in circulation.

${ }^{5}$ Lester, Postlewaite, and Wright (2012) can obtain the partial acceptability of assets in a related setting by assuming a cost to authenticate assets and accept an offer.

${ }^{6}$ This extension offers a bargaining game under private information where both parties enjoy a positive surplus. As a result it would be possible to add an information acquisition choice, as in Lester, Postlewaite, and Wright (2012), according to which a responder could choose to acquire a costly technology to authenticate notes of unknown quality.
} 
Our bargaining setting is a two-dimensional variation of an ultimatum game. ${ }^{7}$ The proposer is endowed with 100 notes and the responder is endowed with 100 widgets. While the widgets have a higher value to the proposer than the responder - thereby generating a motive for trade - the notes have the same terminal value for both agents, which allows them to transfer wealth across subjects and be used as media of exchange. The terminal value of the notes can vary across matches, and the proposer and the responder can be symmetrically, or asymmetrically, informed about these values. The bargaining game instructs the proposer to make a take-it-or-leave-it offer composed of a number of widgets for a number of notes to the responder.

We will study both a complete-information version of this game where the terminal value of the notes is common knowledge, as in Lagos and Wright (2005) or Duffie, Gârleanu, and Pedersen (2005), and a private-information version where responders can produce fake notes at a fixed cost, as in LRW. In the perfect-information baseline, the terminal value of a note is $\$ 0.1$. A widget is worth $\$ 0.1$ to a responder and $\$ 0.2$ to a proposer, which implies a monetary gain from trading a widget equal to $\$ 0.1$. Hence, a proposer and a responder can generate at most $\$ 10$ in a match by exchanging the 100 widgets. We find that the subjects' behaviors in this setting are close to the predictions of the theory: Almost three quarters of all offers are accepted, most trades are individually rational, and are close to the Pareto frontier that would require all 100 notes to be traded. The subjects receive on average almost $60 \%$ of the maximum earnings in a match $(\$ 10)$. The average price of a widget, defined as the number of notes exchanged for a widget, is 1.2 across accepted offers, above the unit price predicted by theory. This difference reflects standard fairness considerations found in the experimental literature on ultimatum games.

We study informational asymmetries by introducing two types of notes, blue and red notes. Blue notes are identical to the notes in the perfect information case; i.e., they pay off $\$ 0.1$ with certainty. In contrast, red notes are worthless, i.e., they pay off $\$ 0$. Responders cannot distinguish genuine from fraudulent notes; i.e., in the experiments they do not observe the color of the notes offered by the proposers. Each proposer is endowed with a $\$ 10$ account that is used to buy either 100 blue notes for $\$ 10$ or to buy 100 red notes at some commonly known dollar cost $C \leq 10$, keeping the remaining $\$(10-C)$ in his account. Across sessions,

\footnotetext{
${ }^{7}$ The decentralized market is directly inspired by the one used in monetary and financial economics (Shi, 1995; Trejos and Wright, 1995; Duffie, Gârleanu, and Pedersen, 2005): Individuals are matched bilaterally and at random, there are gains from trades due to differences in technologies and endowments, and the terms of trade are determined through a simple bargaining protocol. In contrast to these earlier models, we will assume that both commodities (widgets) and money (notes) are divisible as the divisibility of money matters for efficiency in monetary economies (Berentsen and Rocheteau, 2002) and is also key to allow signaling to take place under private information (e.g., Nosal and Wallace, 2007).
} 
we vary the cost of fraud from $\$ 0$ to $\$ 10$ by increments of $\$ 2$.

Key observations emerge from our study. (1) The threat of fraud adversely affects asset liquidity both in terms of the quantities offered in a match (a notion of pledgeability) and acceptance rates. In particular, the threat of fraud reduces the median quantities of notes and widgets offered relative to the perfect information benchmark, and these quantities are higher for higher costs of fraud. Furthermore, fewer offers are accepted under the threat of fraud than under perfect information, and the share of accepted offers increases monotonically with the cost of fraud. (2) The possibility of fraud only generates very small amounts of fraud when $C>0$. In particular, for high costs of fraud $(C>4)$, less than $10 \%$ of offers involve fraudulent notes. This small amount of fraud does not vanish over time. In contrast, when fraud is costless $(C=0)$, about two thirds of offers involve fraudulent notes. (3) There is a signaling mechanism at work to prevent fraud. Blue-note offers tend to involve fewer notes and they tend to be accepted more often. (4) The threat of fraud has an adverse effect on realized gains from trade. Under the threat of fraud, the average earnings in a match can be as low as $\$ 0.3$, which is much lower than the approximate $\$ 6$ gain under perfect information. Moreover, even when fraud is small (less than $10 \%$ of all offers when $C=6$ ) the average earnings are reduced by half. This finding is in accordance with the idea that policies that raise the recognizability of media of exchange, such as the ones typically implemented by Central Banks, are socially worthwhile even when the extent of fraud is limited. ${ }^{8}$

These observations are consistent with the theory developed in the paper. Observation (1) is consistent with the result according to which the threat of fraudulent practices makes asset markets illiquid by reducing the pledgeability and acceptability of assets. We find that the median number of notes offered in a match is in the interval predicted by the theory - between the cost of fraud (expressed in terms of genuine notes) and 100 - for all our treatments. Observation (2) is largely consistent with the no-fraud outcome of the theory according to which, when the cost of fraud is strictly positive, no fraud takes place in equilibrium. In our experiments very small amounts of fraud do occur, and the fraction of fraudulent notes increases as the cost of fraud decreases. This suggests that even small amounts of fraud can provide an indicator of the lack of recognizability of a medium exchange. Observation (2) is also consistent with the prediction that fraud can

\footnotetext{
${ }^{8}$ In terms of policies to improve the recognizability of the currency, the Federal Reserve and the US Bureau of Engraving and Printing redesign notes periodically and they run campaigns to inform the public about the new, distinctive features of notes. See, e.g., http://www.newmoney.gov/.
} 
become significant when counterfeiting notes is costless. Observation (3) confirms the signaling mechanism in the theory according to which the proposer can signal the quality of his notes by retaining a fraction of them, and this fraction increases as fraud becomes cheaper. Finally, in theory the cost of fraud acts as an upper bound on the payment capacity of the subjects and, as a result, it reduces the sizes of the gains from trade that agents can exploit, in accordance with (4). When $C=0$ the subjects should not be able to exploit any gains from trade, which is also in accordance with (4).

One of our concerns was the empirical validity of the equilibrium refinement. First, we can rule out the equilibrium notion used in Nosal and Wallace (2007) since despite the threat of fraud trades do take place. Second, in order to test our equilibrium notion based on the reordering invariance refinement of In and Wright (2011), we studied two alternative timings for the order of the proposers' moves in the bargaining game under private information. In our reference game, the proposer chooses first to purchase blue or red notes, which is not observed by the responder. Following this choice, he is matched with a responder and he extends an offer which can be accepted or rejected. This reference game has a continuum of Perfect Bayesian Nash equilibria because of the difficulty to pin down the responder's beliefs following out-of-equilibrium offers. We replicated our six experiments with varying costs of fraud for the following alternative sequence of moves. First, the proposer chooses an offer to make to the proposer once matched. Second, the proposer chooses to acquire blue or red notes, and simultaneously the responder accepts or rejects the offer. This latter game where observable actions happen first and hidden actions happen last, called the reverse-ordered game, can be studied with subgame perfection as the equilibrium notion, and it is used as a refinement in order to select a subset of the equilibria in the reference game. We find that the outcomes of the reverse-ordered games in terms of offers, acceptability, fraud, and gains from trade, are very similar to those of the original bargaining game. Hence, we conclude that it is a reasonable refinement for bargaining games in decentralized asset markets with private information.

\subsection{Related literature}

The search-theoretic literature on adverse selection in decentralized asset markets with pairwise meetings includes Cuadras-Morato (1994) on the emergence of a commodity money, Velde, Weber, and Wright (1999), and Burdett, Trejos, and Wright (2001) on Gresham's law, and Hopenhayn and Werner (1996) on the 
liquidity structure of asset returns. These papers restrict asset holdings to $\{0,1\}$. The search-theoretic literature on the role of money in the presence of moral hazard problems includes Williamson and Wright (1994), Li (1995), Trejos (1997, 1999), and Berentsen and Rocheteau (2004). In these models, signaling is not possible because money holdings are restricted to $\{0,1\}$ or allocations are restricted to those that are pooling. Banerjee and Maskin (1996) do not restrict asset holdings, but they study the emergence of commodity monies in an environment with Walrasian trading posts. The assumption of price-taking agents rules out the strategic considerations in the pairwise meetings that are the focus of this paper. Lester, Postlewaite, and Wright (2011) describe a model with divisible assets, fiat money, and capital, where the recognizability problem takes the form of claims on capital that can be costlessly counterfeited and that are not accepted unless the buyer of the asset has the technology to authenticate them. Our theory builds on LRW who consider a model of an OTC market where assets are subject to costly fraudulent practices and solve the bargaining game under imperfect information. ${ }^{9}$

Our paper is related to the experimental literature on the role of goods and assets as media of exchange. This literature is reviewed in Duffy (2008, Section 4.1). Brown (1996) and Duffy and Ochs (1999) test the predictions of the search-theoretic model of Kiyotaki and Wright (1989) where the commodity that is used as money emerges endogenously. These studies suggest that the physical properties of commodities (e.g., their storage cost) matter the most for subjects' trading decisions. Duffy and Ochs (2002) study a similar environment where fiat money is added. Our paper emphasizes a different property of monetary assets: their recognizability. Our environment with private information has two types of notes with different terminal values. Similarly, Camera, Noussair, and Tucker (2003) study experiments with fiat money and dividedbearing assets in an an overlapping generations setting to test for rate-of-return dominance patterns. ${ }^{10}$ Camera, Casari, and Bortolotti (2014) study cash versus electronic payments in the lab where electronic is costly to sellers but safe, while cash is costless to sellers but risky. Camera and Casari (2014) and Camera, Casari, and Maria Bigoni (2003) study experiments with intrinsically useless tokens where subjects are allowed to make gifts and investigate the endogenous emergence of monetary exchange. In contrast, we take

\footnotetext{
${ }^{9}$ Additional models of OTC markets with private information include Rocheteau (2011), Camargo and Lester (2013), and Guerrieri and Shimer (2012).

${ }^{10}$ We also ran sessions where subjects can invest in notes with different recognizability properties and rates of return in order to test for the emergence of outcomes featuring rate-of-return dominance. The results of these sessions are not reported in this paper but are available on request.
} 
the institution of money, our notes with positive terminal value, as given. Finally, Duffy and Puzzello (2014) is the first attempt to bring the Lagos-Wright environment in a laboratory setting to test whether subjects use gift exchange rather than monetary exchange.

Our paper is also related to the experimental literature on (ultimatum) bargaining games under private information. Ultimatum games with asymmetric information include Kagel, Kim, and Moser (1996) where players have different information about each other's payoffs, and Miltzkewitz and Nagel (1993) where one subject is uninformed about the size of the gains from trade. Similarly, Forsythe, Kennan, and Sopher (1991) study a bargaining game where agents have asymmetric information about the gains from trade and interpret strikes as the failures of the bargainers to agree on a division of the surplus. For a review of experimental work on bargaining under incomplete information, see Camerer (2003, Section 4.3).

Closer to what we do, Forsythe, Lundholm, Rietz (1999) consider an experiment where subjects are divided between buyers and sellers of assets, sellers hold assets of unknown quality, and buyers make offers that sellers can accept or reject. In contrast, in our model gains from trade arise because subjects can exchange a good of homogenous quality that they value differently, and we emphasize the role of an asset that is commonly valued across subjects as a medium of exchange. The private information problem takes the form of costly fraud, and we let the uninformed party make an offer, which opens up the possibility for signaling.

\section{OTC bargaining game under perfect information}

Our experiment aims to describe an OTC market where individuals are matched bilaterally and at random and bargain over the terms of trade. ${ }^{11}$ In each match, there are gains from trade due to different endowments and production technologies, and there is an asset playing the role of a medium of exchange. The bargaining game is a simple ultimatum game where the asset holder makes the offer. ${ }^{12}$ As shown in the monetary literature, under symmetric information this bargaining protocol maximizes the liquidity value of the asset. ${ }^{13}$

\footnotetext{
${ }^{11}$ In reality this OTC market can be a market for financial assets, as described in Duffie, Garleanu, and Pedersen (2005), or a decentralized market for goods and services, as in Shi (1995), Trejos and Wright (1995).

${ }^{12}$ Our bargaining game differs from the standard ultimatum game in that offers are two-dimensional and realized gains from trade vary with the offers.

${ }^{13}$ For a review of the relevant search-theoretic literature on monetary exchange, see Rupert, Schindler, Schevchenko, and Wright (2000).
} 
The two players in the bargaining game are called Proposer and Responder. ${ }^{14}$ The proposer is endowed with 100 units of a divisible asset called notes. These notes pay off a certain amount of a numéraire good at the end of the period. A key property of notes is that they yield the same payoff irrespective of who is holding them; i.e., their value is common to all participants. ${ }^{15}$ However, notes might come in different qualities; i.e., they differ in the amount of the numéraire good that they pay off at the end of the period. Later on, we introduce private information, by assuming that individuals may have different information about the quality of these notes.

A responder is endowed with 100 units of an intermediate good called a widget. Proposers and responders have access to different technologies to produce the numéraire good from widgets. A proposer can produce two units of the numéraire good per widget, while a responder can only produce one unit of the numéraire good per widget. This difference in productivities generates gains from trade for proposers and responders. ${ }^{16}$

As a benchmark we consider the case where notes are homogenous and the proposer and the responder have perfect information about the terminal value of notes. The trading mechanism is such that the proposer (the note holder) makes a take-it-or-leave-it offer to the responder. An offer is a pair, $(\omega, n) \in[0,100]^{2}$, where $\omega$ is the quantity of widgets received by the proposer from the responder and $n$ is the quantity of notes delivered by the proposer to the responder. In the theoretical analysis we assume that all objects are divisible. ${ }^{17}$

We assume that proposers and responders are risk-neutral. This approximation is justified under the

\footnotetext{
${ }^{14}$ In the context of the Shi-Trejos-Wright model, the proposer would be the buyer (of goods and services) and the responder would be the seller. In the context of the Duffie-Garleanu-Pedersen model, the proposer would be the investor with a high valuation for the (financial) asset, while the responder would be the investor with the low valuation for the asset.

${ }^{15}$ Engineer and Shi $(1998,2001)$ in environments with indivisible money and Berentsen and Rocheteau (2003) in an environment with divisible money emphasize the role of money to transfer utility perfectly across agents. In these models, fiat money allows traders to separate the decisions of how much to produce and how to split the resulting total surplus. Jacquet and Tan (2012) use a related argument to explain why fiat money has a higher liquidity than Lucas trees. In their model, Lucas trees that yield state-dependent dividends are valued differently by agents with different hedging needs. It follows that agents have an endogenous preference for money as a means of payment, because in contrast to Lucas trees they are valued equally by all agents.

${ }^{16}$ In the context of the Shi-Trejos-Wright model, the utility of the buyer from consuming $q$ units of goods would be $u(q)=2 q$, and the (opportunity) cost of the seller would be $c(q)=q$. Our model differs from the Shi-Trejos-Wright model in that the surplus of a match, $u(q)-c(q)$, is not strictly concave. However, the gains from trade are bounded above due to the finite endowment of the Responder. One can also interpret our assumptions in the context of the Duffie-Garleanu-Pedersen model. One can think of the widget as an asset that has a terminal value equal to 2 . The Responder has no cost from holding the asset while the Proposer incurs a cost equal to one, for example, because of liquidity needs or hedging reasons.

${ }^{17}$ The fact that both money (notes) and goods (widgets) are divisible contrasts with the setting in Shi (1995) and Trejos and Wright (1995) where money is in $\{0,1\}$ and that in Duffie, Garleanu, and Pedersen (2005) where the asset is in $\{0,1\}$. In this regard, our model is closer to the new generation of monetary models of Shi (1997), and Lagos and Wright (2005), and the model of an OTC financial market of Lagos and Rocheteau (2009). In the experiments, the players have to choose integers in $\{0, \ldots, 100\}$.
} 
expected utility paradigm when stakes are small (e.g., Arrow, 1971, p.100). ${ }^{18}$ For proposers and responders alike, one note buys one unit of the numéraire good, and one unit of the numéraire good yields one utile. If a proposer offers the trade $(\omega, n)$ and if the trade is accepted, he receives $\omega$ widgets and keeps $100-n$ notes. Accordingly, his payoff (in terms of the numéraire) is $U_{P}=2 \omega+100-n$. For the same trade, the responder keeps $100-\omega$ widgets and receives $n$ notes. Accordingly, his payoff is $U_{R}=100-\omega+n$.

Throughout this paper, we want to assess whether the trades that we observe in the laboratory satisfy basic requirements in terms of individual rationality, Pareto efficiency, and whether they accord with some standard equilibrium notion (e.g., subgame perfection). When we turn to the equilibrium, we will extend our model to introduce fairness considerations in the bargaining so that the predictions of the model are closer to the data.

Individual rationality. For a proposer, a trade that yields a positive surplus satisfies $2 \omega+100-n \geq 100$; i.e., $S_{P} \equiv 2 \omega-n \geq 0$. Accordingly, the set of individual rational trades for a proposer is

$$
P \equiv\left\{(\omega, n) \in[0,100]^{2}: 2 \omega-n \geq 0\right\}
$$

For a responder, a trade that yields a positive surplus satisfies $100-\omega+n \geq 100$; i.e., $S_{R} \equiv n-\omega \geq 0$. Accordingly, the set of individual rational trades for a responder is

$$
R \equiv\left\{(\omega, n) \in[0,100]^{2}: n-\omega \geq 0\right\}
$$

The set of feasible, individual rational trades is then $S=P \cap R$; i.e.,

$$
S \equiv\left\{(\omega, n) \in[0,100]^{2}: 2 \omega \geq n \geq \omega\right\}
$$

The set $S$ is depicted in Figure 1 as the grey-shaded area. The curve labelled $I R_{P}$ is the set of trades that yields zero surplus to the proposer; i.e., $2 \omega=n$, and the one labelled $I R_{R}$ is the set of trades that yield zero surplus to the responder; i.e., $\omega=n$. The first key benchmark for the experiments will be to assess whether the trades are in the set $S$, meaning that our subjects satisfy basic rationality assumptions.

\footnotetext{
${ }^{18}$ For a critical discussion of this assumption, see Rabin (2000).
} 


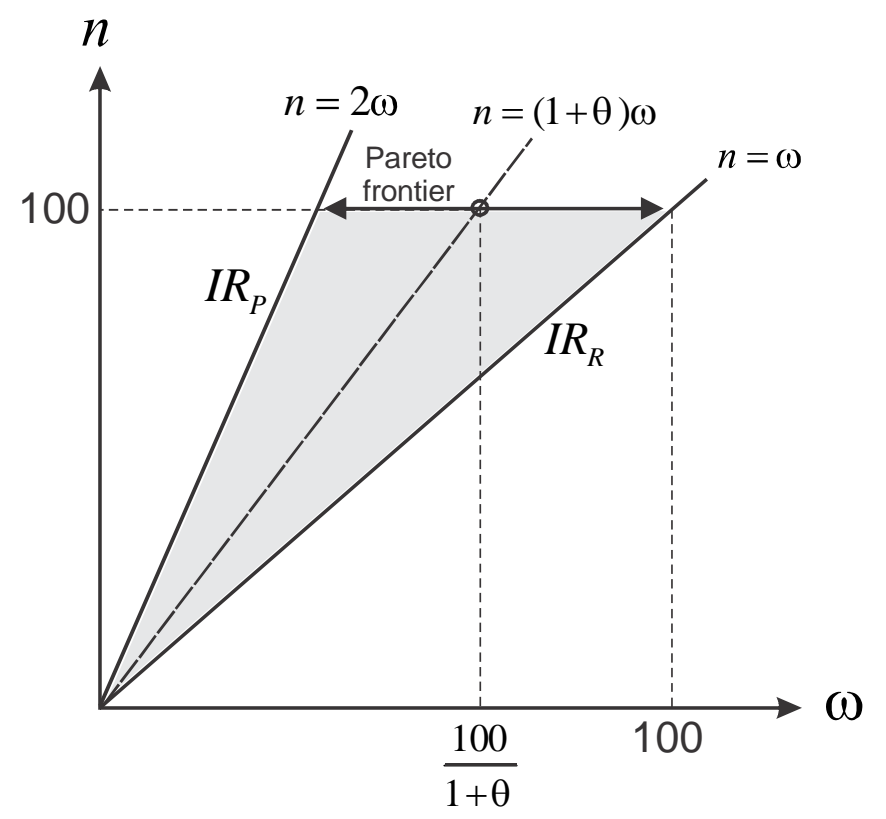

Figure 1: Bargaining game under complete information

Pareto efficiency. Our second key benchmark will be to assess whether the trades are Pareto-efficient. ${ }^{19}$ The Pareto frontier associated with this bargaining problem solves

$$
\mathcal{S}_{P}=\max _{\omega, n}(2 \omega-n) \text { s.t. } n-\omega=\mathcal{S}_{R}
$$

and $(\omega, n) \in[0,100]^{2}$. Pareto-efficient trades are such that the proposer offers all his notes $(n=100)$ and asks for $\omega \in[50,100]$ widgets. The equation for the Pareto frontier in the utility space is $\mathcal{S}_{P}+2 \mathcal{S}_{R}=100$. See the top quadrant of Figure 2. So the proposer obtains a surplus equal at most to 100, while the maximum surplus of the responder is $50 .{ }^{20}$ The set of Pareto-efficient offers is represented in the bottom quadrant of Figure 2.

Equilibrium under fairness A third benchmark is to assess whether our subjects are able to coordinate on a subgame perfect equilibrium (SPE). In order to obtain a better representation of the experimental data,

\footnotetext{
${ }^{19}$ This property of the allocation corresponds to the pairwise core requirement in the mechanism design literature in monetary theory. See, e.g., Hu, Kennan, and Wallace (2009).

${ }^{20}$ Notice that if proposers were not constrained by the number of notes they hold, e.g., they hold at least 200 notes, then the equation for the Pareto frontier would be $\mathcal{S}_{R}+\mathcal{S}_{R}=100$ and all Pareto-efficient trades would be such that $\omega=100$.
} 


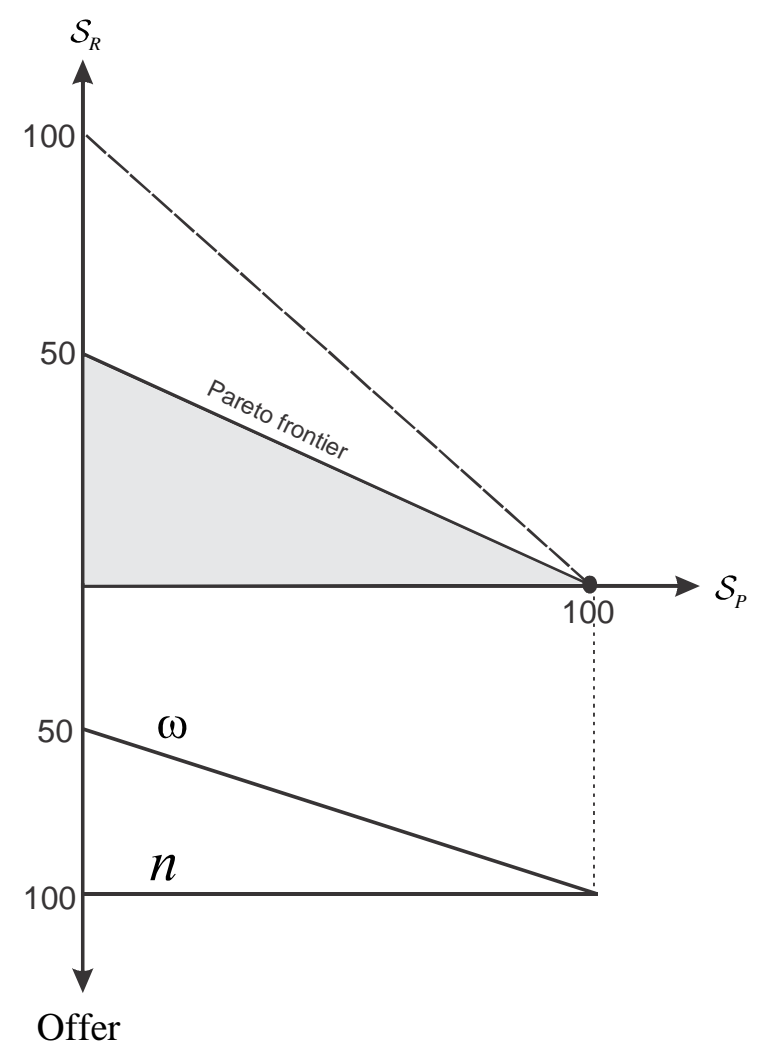

Figure 2: Pareto frontier of the bargaining set

we extend our model to allow responders to value fairness. More precisely, we assume that the responder suffers a utility loss equal to $\theta /(1-\theta)$ times the surplus of the proposer, $\mathcal{S}_{P} \equiv 2 \omega-n \geq 0$, where $\theta \in[0,1]$. The motivation function of the responder becomes

$$
\hat{U}_{R}=\overbrace{U_{R}}^{\text {payoff }}-\overbrace{\frac{\theta}{1-\theta} \mathcal{S}_{P}}^{\text {fairness loss }}=100+\frac{n-(\theta+1) \omega}{1-\theta} .
$$

Hence, the behavioral surplus of the responder is

$$
\hat{S}_{R} \equiv \mathcal{S}_{R}-\frac{\theta}{1-\theta} \mathcal{S}_{P}
$$

In order words, in order to evaluate his own surplus the responder adopts as a reference point the surplus of the proposed scaled by some constant. For a responder to agree to a trade, $\hat{S}_{R} \geq 0$, his own surplus, $\mathcal{S}_{R} \equiv n-\omega$, must be larger than $\theta /(1-\theta)$ times the surplus of the proposer. 
Behavioral Assumption. Responders only accept offers that satisfy $\mathcal{S}_{R} \geq \frac{\theta}{1-\theta} \mathcal{S}_{P}$.

We directly incorporate this notion of fairness for three reasons. ${ }^{21}$ First, our formulation is equivalent to Kalai's (1977) proportional bargaining solution, which has been used extensively in the monetary literature. Second, a large body of experimental work finds that a concern for fairness is often present in bilateral bargaining. ${ }^{22}$ So incorporating fairness into our bargaining framework will help us to reconcile the theory with our experimental evidence. Third, the generalized model with fairness encompasses the standard model described so far, where $\theta=0$. So, adding fairness will provide us with a wider range of predictions. Moreover, we will see that fairness can interact with informational frictions in interesting ways. For the theory, we will assume that $\theta$ is common knowledge in a match.

A utility maximizing proposer chooses a trade $(\omega, n)$ that maximizes his surplus, $2 \omega-n$, subject to the constraint that the offer is fair to the responder; i.e., it is such that $S_{R} \geq \frac{\theta}{1-\theta} S_{P}$. Therefore, the proposer solves the following problem:

$$
\max _{\omega, n}(2 \omega-n) \text { s.t. } n-\omega \geq \frac{\theta}{1-\theta}(2 \omega-n)
$$

and $(\omega, n) \in[0,100]^{2}$. The solution is $n=100$ and $\omega=100 /(1+\theta)$. It is marked by a circle on the Pareto frontier in Figure 1. The proposer's surplus is equal to $(1-\theta) \times 100$, while the responder's surplus is $\theta \times 100$. As $\theta$ varies on $[0,1]$, the outcome describes the whole Pareto frontier. The data from the experiments will allow us to estimate $\theta$.

In what follows, we formulate Hypothesis 1, which contains the key predictions from the theory.

Hypothesis 1 (Symmetric Information) Under symmetric information (SI), proposers will offer 100 notes and ask for a number of widgets between 50 and 100.

\footnotetext{
${ }^{21}$ The direct incorporation of a preference for fairness is somewhat controversial among the profession. Here, we have chosen to do it in a way that is consistent with both the monetary literature, where the proportional bargaining solution has been used extensively (e.g., Aruoba, Rocheteau, and Waller, 2007) and the experimental literature. Two well-known ways to incorporate fairness in bargaining models are presented by Bolten and Ockenfels (2000) and by Fehr and Schmidt (1999). Bolton and Ockenfels (2000) make an assumption related to ours where an agent's share in the total payoff of the game is an argument of the player's motivation function. Similarly, our assumption is consistent with the notion of equity aversion of Fehr and Schmidt (1999) if we set $\beta_{i}=0$ (agents only care about inequity that is to their material disadvantage).

${ }^{22}$ See Roth (1995) for a review of the bargaining literature and a discussion of studies that test this fairness hypothesis. In short, preferences for fairness are often present, but comparison of results from Ultimatum Game and Dictator games reveal that other factors such as negative reciprocity among responders and fear of negative reciprocity among proposers is also present. For simplicity, our formulation accounts for fairness alone.
} 


\section{OTC bargaining under the threat of fraud}

We now consider a game based on LRW where the quality of the notes is chosen by the proposer. This model captures a situation where a con-artist may produce counterfeit notes or where a financial institution may originate and securitize bad loans that are sold afterwards to other investors. ${ }^{23}$ At the beginning of the game, the proposer has the choice between purchasing 100 blue (genuine) notes at the unit cost of one in terms of the numéraire or 100 red (counterfeit) notes at a total cost, $C .{ }^{24}$ For proposers and responders alike, at the end of the game, one blue note buys one unit of the numéraire good, and, as above, one unit of the numéraire good yields one utile. Red notes are worthless, and responders cannot distinguish blue from red notes. To analyze this bargaining game with hidden actions, we adopt the methodology from In and Wright (2011) for signaling games with endogenous types. ${ }^{25}$ According to this methodology, one can look at a strategically equivalent game, the so-called reverse-ordered game. In this game, all observable moves are made first. In our context, in the reverse-ordered game the proposer chooses his offer first, and then he decides whether to acquire blue or red notes in order to execute his offer if it is accepted. The advantage of this methodology is that following an offer, there is a proper subgame that can be easily analyzed. ${ }^{26}$

To see this, consider an arbitrary offer, $(\omega, n)$, and suppose that this offer is accepted with probability $p$. Let $\eta$ denote the probability that the proposer acquires blue notes after offering $(\omega, n)$. It satisfies the following best-response function:

$$
-100+p(2 \omega+100-n)+(1-p) 100\left\{\begin{array} { l } 
{ > } \\
{ = - C + p 2 \omega \Rightarrow \eta } \\
{ < }
\end{array} \left\{\begin{array}{c}
=1 \\
\in[0,1] \\
=0
\end{array} .\right.\right.
$$

The left side of (6) is the proposer's expected payoff if he chooses to acquire blue notes: He pays 100 to purchase 100 blue notes, with probability $p$ his offer is accepted, in which case he receives $\omega$ widgets and keeps $100-n$ notes, and with probability $1-p$ the offer is rejected, in which case the proposer ends up with

\footnotetext{
${ }^{23}$ For examples of fraud on media of exchange, see Li, Rocheteau, and Weill (2012).

${ }^{24}$ As shown in Li, Rocheteau, and Weill (2012) in the presence of a fixed cost of fraud we can restrict strategies with no loss in generality so that either all notes are blue or all are red but intermediate choices are ruled out.

${ }^{25}$ We cannot apply standard refinements of signaling games, such as the intuitive criterion, because "types" are chosen in the initial stage instead of being determined by Nature. We instead apply the reordering invariance refinement of In and Wright (2011), based on the invariance condition of strategic stability from Kohlberg and Mertens (1986), which requires that the solution of a game should also be the solution of any game with the same reduced normal form. From a normative viewpoint, this refinement has the appealing property of selecting an equilibrium of the original game that yields the highest payoff to the proposer, the agent making the offer.

${ }^{26}$ This key feature of the reverse-ordered game allows us to pin down beliefs following all out-of-equilibrium offers in a logically consistent way, and it improves tractability dramatically as subgame perfection becomes sufficient to solve the game.
} 
100 blue notes. The right hand side of (6) is the expected payoff of the proposer if he acquires red notes: The cost of red notes is $C$, and with probability $p$ the offer is accepted, in which case he receives $\omega$ widgets. The best-response function (6) can be rewritten as

$$
p n\left\{\begin{array}{l}
< \\
=C \Rightarrow \eta\left\{\begin{array}{c}
=1 \\
>
\end{array}\right][0,1] . \\
=0
\end{array}\right.
$$

The condition, $p n \leq C$, for the accumulation of blue notes can be interpreted as a liquidity or resalability constraint. ${ }^{27}$ It states that for the proposer not to have incentives to acquire fraudulent assets, the expected value of the assets that are exchanged in a match has an upper bound, which is given by the cost to produce fraudulent assets.

The responder accepts an offer $(\omega, n)$ if $\mathbb{E}\left[\hat{U}_{R}\right] \geq 100$, where the expectation is with respect to the terminal value of the notes. Consequently, an offer is acceptable if $\mathbb{E}\left[\mathcal{S}_{R}\right] \geq \frac{\theta}{1-\theta} \mathbb{E}\left[\mathcal{S}_{P}\right]$, which is a generalization of our previous behavioral assumption to the case where the quality of the notes is uncertain. ${ }^{28}$ If the responder believes the notes are genuine with probability $\eta$, then his best-response is

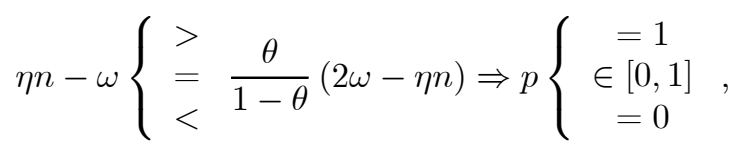

since $\eta n$ is the expected value of the notes, and $\omega$ is the cost of giving up $\omega$ widgets. This best response can be re-expressed as

$$
\eta n\left\{\begin{array}{l}
> \\
\left.=(1+\theta) \omega \Rightarrow p\left\{\begin{array}{c}
=1 \\
\in \\
<
\end{array}\right] .1\right] . \\
=0
\end{array}\right.
$$

Based on the best-response functions (7) and (8), the Nash equilibria of the subgame following an offer $(\omega, n)$ in the reserve-ordered game are represented in Figure 3. If the proposer's offer is such that $(1+\theta) \omega<$ $n<C$, then the unique Nash equilibrium in the subgame following that offer is such that $p=1$ and $\eta=1$. Intuitively, if $n<C$, then from (7), it is optimal to set $\eta=1$. If $\eta=1$, then from (8) it is optimal to accept the offer; i.e., to set $p=1$. Thus, if the value of the offered blue notes is less than the cost of fraud and if the responder's surplus is fair, then there is no fraud and the offer is accepted. If the proposer's offer is such that $n>C$ and $n>(1+\theta) \omega$, then the equilibrium of the subgame following that offer involves mixed strategies.

\footnotetext{
${ }^{27}$ If $p n>C$, then the proposer acquires red notes with certainty; i.e., $\eta=0$.

${ }^{28}$ We kept the formalization of the fairness motive identical to the one under complete information. In particular, we treated the cost of fraud as sunk when measuring the expected surplus of the proposer. As before, the OTC bargaining game without fairness consideration is obtained by setting $\theta=0$. This corresponds to the case studied in Li, Rocheteau, and Weill (2012).
} 


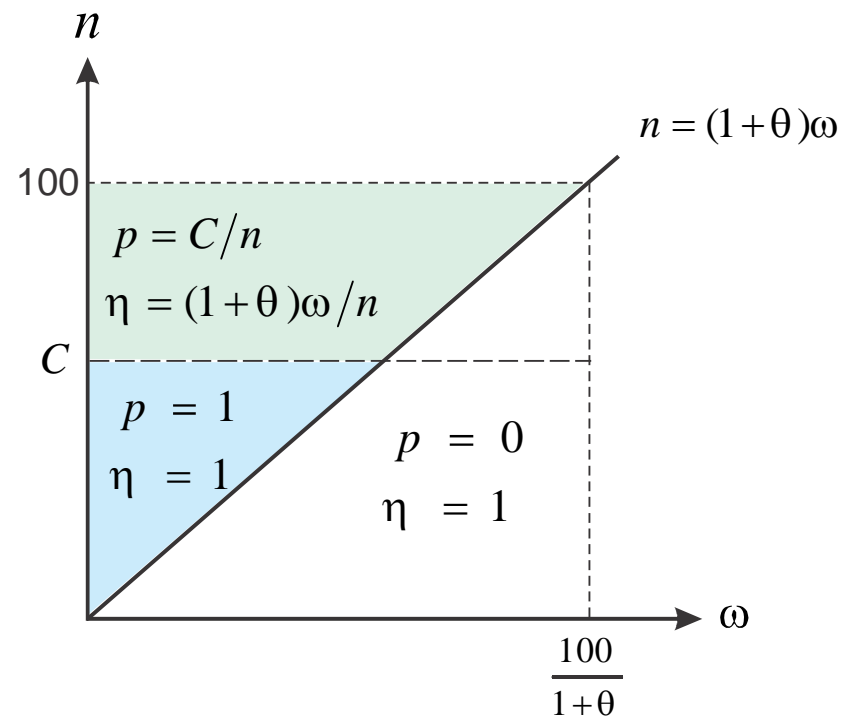

Figure 3: Nash equilibria following an offer $(\omega, n)$ in the reverse-ordered game.

In this mixed-strategy equilibrium, the responder is indifferent between accepting or rejecting the offer and the proposer is indifferent between purchasing blue notes or red ones. In such a mixed-strategy equilibrium, $p=C / n$ and $\eta=(1+\theta) \omega / n$. Intuitively, if $n>C$, then from (7), in order to induce the proposer to choose blue notes, we must have $p<1$. Furthermore, if $n>(1+\theta) \omega$, then from (8), in order to induce the responder to choose $p<1$, we must have $\eta<1$. An interesting property of this equilibrium is that as the number of notes offered increases, the probability of acceptance decreases and the probability of fraud increases. The results also show that fairness considerations interact with the private information friction. First, as $\theta$ increases, the set of offers associated with a positive level of trade shrinks. Second, in terms of the comparative statics of mixed-strategy equilibria, as $\theta$ increases, the probability of fraud decreases (i.e., $\eta$ increases). Intuitively, if the responder values fairness more, then the average quality of notes must increase in order to keep him indifferent between accepting or rejecting an offer.

Given the Nash equilibria of the subgames following all possible offers, the proposer at the beginning of the reverse-ordered game tree chooses the offer that maximizes his expected payoff. An optimal offer will not involve counterfeiting (unless $C=0$ ). To see this, suppose that the offer, $(\omega, n)$, corresponds to a mixed-strategy equilibrium with $\eta=(1+\theta) \omega / n<1$ and $p=C / n<1$. The proposer could deviate and make 
an alternative offer, $\left(\omega^{\prime}, n^{\prime}\right)$, with $\omega^{\prime}=\omega$, but $n^{\prime}<n$. Following such an offer, the Nash equilibrium would be such that $\eta^{\prime}>\eta$ and $p^{\prime}>p$, and hence the proposer would be better off since his payoff is $-C+p(2 \omega) .{ }^{29}$ The intuition for this no-counterfeiting result is that counterfeiting in equilibrium can never benefit the proposer given that it is fully anticipated by the responder. In fact, counterfeiting would harm the proposer by reducing his payment capacity. Using that $\eta=1$, the optimal offer of the proposer solves

$$
\max _{\omega, n, p} p(2 \omega-n) \text { s.t. } n-(1+\theta) \omega=0 \text { and } p n \leq C .
$$

The proposer maximizes his expected surplus subject to two constraints. The first constraint is the participation constraint of the responder, where it is assumed that the responder believes that the notes are blue. The second constraint is the resalability constraint (7).

If $C<100$, the resalability constraint is binding. In this case, the solution is $p n=C, n=(1+\theta) \omega$, $p \in\left[\frac{C}{100}, 1\right]$. This means that $(1+\theta) \omega=n \in[C, 100]$ as indicated in Figure 4. If $n=(1+\theta) \omega=C$, the offer is accepted with probability one, but the proposer cannot purchase more $C /(1+\theta)$ widgets. The proposer can offer to sell a larger number of notes, but then his offer will be rejected with positive probability; i.e., $p<1$. The result that, because of the threat of fraud, some offers can be rejected in equilibrium is new relative to LRW. The model, therefore, captures the notion that large note offers are less liquid and it shows that the private information problem affects both the intensive margin of trade, $(\omega, n)$, and the extensive margin, the fraction of matches where a positive trade takes place. ${ }^{30}$

Across all the optimal offers that solve (10), the expected surplus of the proposer is

$$
\mathbb{E}\left[\mathcal{S}_{P}\right]=p(2 \omega-n)=\left(2 \frac{\omega}{n}-1\right) p n
$$

Using that $p n=C$, from the no-counterfeiting constraint, and $\omega / n=1 /(1+\theta)$, from the responder's participation constraint, the expected surplus of the proposer is $\mathbb{E}\left[\mathcal{S}_{P}\right]=(1-\theta) C /(1+\theta)$. By a similar reasoning, the surplus of the responder is $\mathbb{E}\left[\mathcal{S}_{R}\right]=\theta C /(1+\theta)$.

We illustrate the set of optimal offers in Figure 4. The grey shaded area is the set of offers that satisfy the proposer's individual rationality constraint, the responder's fairness constraint, and the proposer's incentive

\footnotetext{
${ }^{29} \mathrm{See} \mathrm{Li}$, Rocheteau, and Weill (2012) for a detailed proof.

${ }^{30}$ This property of our model differs from the version in Li, Rocheteau and Weill (2012, Proposition 1) under strictly concave utility for the proposer in which equilibrium offers are always accepted with probability one.
} 


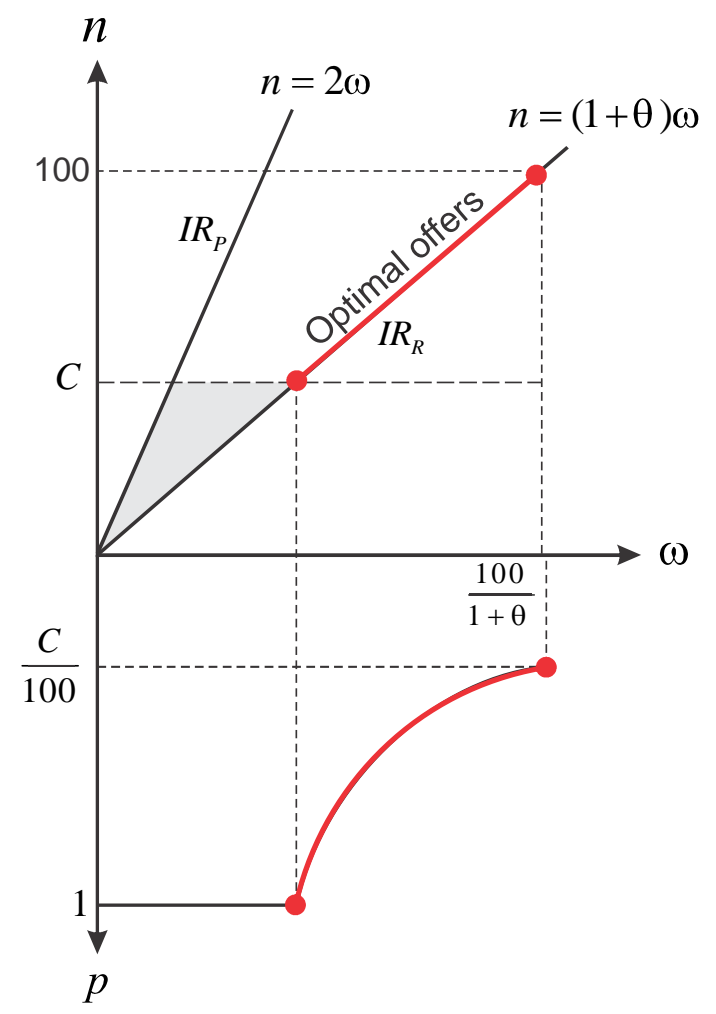

Figure 4: Bargaining under the threat of fraud

compatibility constraint when $p=1$. Among this set of incentive-feasible offers, the preferred one of the proposer is $n=(1+\theta) \omega=C$. There are larger offers, $n=(1+\theta) \omega>C$, that are payoff equivalent, but they are rejected with a positive probability, $p<1$. For instance, the proposer could make the perfect information offer, $n=(1+\theta) \omega=100$, but in this case the offer would be accepted with probability $p=\frac{C}{100}<1$.

Finally, if fraud is costless, $C=0$, then no trade takes place in equilibrium, and proposers are indifferent between acquiring blue notes or red notes, $\eta \in[0,1]$. Therefore, in this limiting case, fraud can emerge in equilibrium, but all offers should get rejected.

Hypothesis 2 (OTC bargaining game under the threat of fraud)

1. If $C=0$, fraud can occur, but no offer should be accepted.

2. If $C>0$, then: 
(a) No fraud takes place $(\eta=1)$.

(b) The minimum number of notes offered is $C(n \geq C)$.

(c) Offers such that $n \geq C$ are accepted with probability $p=C / n$.

(d) The number of widgets offered is $\omega=n /(1+\theta)$.

\section{Experiment design}

We conducted multiple experiment sessions at the UC Irvine Experimental Social Science Laboratory with 360 students as human subjects. ${ }^{31}$ Students learned of experiments via posted advertisements and email announcements, and they registered to be subjects in the laboratory's subject pool through an online registration system. Days before each experiment session, an email was sent to a random selection of subjects in the pool notifying them of our upcoming session. Interested students then signed up for a specific session on the subject pool web site. Those who signed up received a reminder email about the session the day before it was conducted. Subjects received a $\$ 7$ show-up payment plus earnings based on the decisions made, with final take-home amounts rounded up to the nearest quarter. To compute these earnings, we assign $\$ 0.1$ for each blue note held by a proposer or a responder, and $\$ 0.2$ for each widget held by a proposer and $\$ 0.1$ for each widget held by a responder. Red notes have no value. The average take-home amount across all sessions was about $\$ 17$ for about 75 minutes of participation.

As shown in Table 1, these sessions are identified under the labels SI, MH, and RMH. We conducted a single, baseline session, labeled SI for Symmetrically Informed, in which both parties have common knowledge that all notes are blue. There are six Moral Hazard sessions, labelled $\mathrm{MH}_{10}, \ldots, \mathrm{MH}_{0}$, where the index indicates the costs in $\$$ of acquiring 100 red notes, $C \in\{10,8,6,4,2,0\}$. (It is worth remembering that the cost of fraud in the theory section is expressed in terms of the terminal value of genuine notes. If we adopt this unit of measure it is equal to $10 C$.) In each of these sessions, proposers have to decide whether to buy 100 blue notes for $\$ 10$ or 100 red notes for $\$ C$. Reverse-ordered Moral Hazard sessions are similarly labeled $\mathrm{RMH}_{10}, \ldots, \mathrm{RMH}_{0}$.

\footnotetext{
${ }^{31}$ We here report results from thirteen sessions. Additional sessions were also conducted, but are not reported here. For instance, we ran six sessions with unrepeated one-shot games and found results roughly consistent with the results presented here. We also conducted sessions with symmetrically uninformed actors and sessions where the value of notes although privately known by the proposer is chosen by Nature. Some of these sessions are discussed below when interpreting our results.
} 
TABle 1: Experiment SESSiOns

\begin{tabular}{|c|c|c|c|c|}
\hline Information Setting & Session Identifier & Treatment Details & \# Subj. & Avg. $\$^{a}$ \\
\hline Symmetric Informed & $\mathrm{SI}$ & Baseline, $100 \%$ blue notes & 30 & $\$ 20.08$ \\
\hline \multirow{4}{*}{\begin{tabular}{c} 
Moral Hazard \\
\cline { 1 - 5 }
\end{tabular}} & $\mathrm{MH}_{10}$ & Red notes cost $\$ 10$ & 34 & $\$ 16.60$ \\
\cline { 2 - 5 } & $\mathrm{MH}_{8}$ & Red notes cost $\$ 8$ & 30 & $\$ 17.17$ \\
\cline { 2 - 5 } & $\mathrm{MH}_{6}$ & Red notes cost $\$ 6$ & 22 & $\$ 18.43$ \\
\cline { 2 - 5 } & $\mathrm{MH}_{4}$ & Red notes cost $\$ 4$ & 30 & $\$ 16.55$ \\
\cline { 2 - 5 } & $\mathrm{MH}_{2}$ & Red notes cost $\$ 2$ & 32 & $\$ 16.88$ \\
\hline \multirow{5}{*}{$\begin{array}{c}\text { Reverse-ordered } \\
\text { Moral Hazard }\end{array}$} & $\mathrm{MH}_{0}$ & Red notes cost $\$ 0$ & 20 & $\$ 17.30$ \\
\cline { 2 - 5 } & $\mathrm{RMH}_{10}$ & Red notes cost $\$ 10$ & 30 & $\$ 18.90$ \\
\cline { 2 - 5 } & $\mathrm{RMH}_{8}$ & Red notes cost $\$ 8$ & 30 & $\$ 18.77$ \\
\cline { 2 - 5 } & $\mathrm{RMH}_{6}$ & Red notes cost $\$ 6$ & 22 & $\$ 17.89$ \\
\cline { 2 - 5 } & $\mathrm{RMH}_{4}$ & Red notes cost $\$ 4$ & 30 & $\$ 16.93$ \\
\cline { 2 - 5 } & $\mathrm{RMH}_{2}$ & Red notes cost $\$ 2$ & 28 & $\$ 16.91$ \\
\hline
\end{tabular}

To facilitate the experiment's management, instruction, and data collection, we used the z-Tree software package (Fischbacher 2007). Each session consisted of three stages: instruction, decision making, and questionnaire. During the instruction stage, subjects read about the decision-making scenario and answered questions to test their comprehension about the payoffs associated with the different decision-making roles. After answering each question, the subject is told whether his or her answer was correct and is given a complete explanation of the correct answer. The instructions were identical across sessions except for changes in the description and questions that correspond to the specifics of the treatment. ${ }^{32}$

The decision-making stage consists of twenty rounds of one-shot interactions. ${ }^{33}$ Subjects are first randomly assigned to proposer and responder roles, which they maintain during all twenty rounds. They are then randomly and anonymously matched into the first one-shot interaction. After completion of the first round, the subjects were rematched randomly and anonymously for the second round, and so on for the rest of the twenty rounds. ${ }^{34}$ As typical in other experimental studies, behavior tends to converge after the early rounds. This fact can be seen in Figure 5, which charts mean offer characteristics and acceptance rates during each session's duration. Our analysis below thus drops the first five rounds, and throughout this

\footnotetext{
${ }^{32}$ See the Supplemental Appendix for instructions for a representative session.

${ }^{33}$ Our setting extends the standard ultimatum game to a two-dimensional setting but where each party is given an initial endowment rather than just the proposer. The take-it-or-leave-it offers are standard in ultimatum games, though a literature with free-form, face-to-face discussion exists. See, for example, earlier experiments on Coasian bargaining, some of which also include risk or uncertainty (e.g., Hoffman and Spitzer, 1982; Harrison and McKee 1985).

${ }^{34}$ Our matching procedure does not rule out the possibility that that the two same subjects are matched more than once over the twenty rounds. Alternatively, we could have implemented a "perfect stranger matching" that would avoid with certainty repeated interactions. Because outcomes under random matching and perfect-stranger matching are typically quite similar and because the number of our subjects was large enough to make the chance of repeated matches quite small, we chose the former.
} 
paper, we report statistics from the last 15 rounds.

At the end of the last round, the computer randomly selects one round, and all subjects are paid according to the decisions for that round. This one-round payment mechanism is widely used in experiments to best implement a one-shot decision. To ensure that each session ends on time, decisions were made with explicit time constraints. Proposers were given 120 seconds in rounds 1-3, and 45 seconds in all other rounds, or else the computer would make an offer of 0 notes for 0 widgets. Responders were given 60 seconds in rounds 1-3, and 30 seconds in all other rounds, or else the computer would reject the offer. This time constraint was never binding for responders, but the time limit was reached among some proposers. See the discussion below.

The questionnaire asks the subject to report personal information, such as sex, race, and academic major. A breakdown of self-reported subject characteristics by session as well as other statistics on subjects' behavior can be found in a supplement appendix available from the authors. The questionnaire data reveal a wide distribution of subjects, with higher proportions of female students, engineering and biological sciences students than in the university's undergraduate population.

\section{$5 \quad$ Experiment results}

We first present the experimental evidence on the outcomes of the OTC bargaining game under perfect information. The evidence is largely consistent with the theory once fairness considerations are taken into account. We then review the results for the OTC bargaining game with private information and the threat of fraud. Due to space considerations, we can only present the most important data analysis and findings. A supplement appendix, available from the authors, contains additional analyses and figures.

\subsection{Perfect information (SI)}

The SI session provides a benchmark relative to which we can assess the effects of informational frictions on market outcomes. We organize the presentation of the experimental evidence through a sequence of results characterizing the terms of the proposers' offers, responders' acceptance rates, and earnings. We start with a description of proposers' offers both in terms of quantities and prices.

RESULT \#1 (Offers under perfect information). Under perfect information, the median number of 

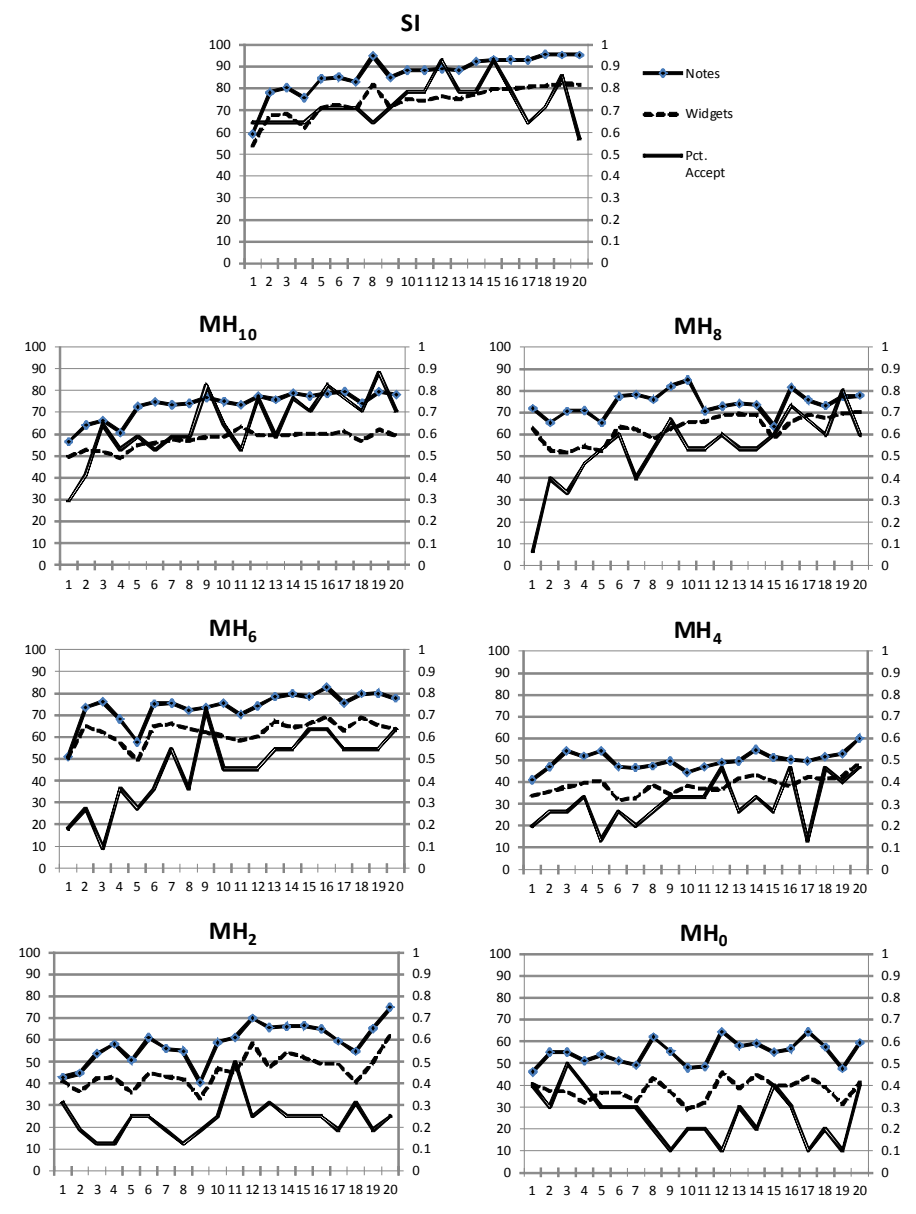

Figure 5: Mean notes offered, widgets requested, and acceptance rate by round, SI and MH sessions notes offered is 100 . The median offer price of a widget in terms of notes $(n / \omega)$ is larger than one.

The average number of notes offered is 87 out of 100; the median notes offered is 100 with $63 \%$ of proposers offering the full 100 notes. ${ }^{35}$ The fact that the median subject offers 100 notes is consistent with the assumption of Pareto-efficient trades. The median (average) widgets requested is 85 (74), well within the $[50,100]$ range of Pareto-efficient trades. As seen in Figure 5, offers come closer to the predicted behavior as the experiment progresses, suggesting that subjects are learning to make better offers. The median offer

\footnotetext{
${ }^{35}$ Given that the endowment of notes is 100 , the possible offers have a downward bias relative to the subgame-perfect equilibrium offer of 100 notes for 100 widgets.
} 
price is 1.14 , the median price of accepted offers is 1.20 , and the median price of rejected offers is 1.11. Every accepted offer has a price greater than or equal to 1, and accepted offers have, on average, higher prices than rejected offers (see Figure 8).

RESULT \#2 (Acceptance rate under perfect information). Under perfect information more than $76 \%$ of all offers are accepted.

The fraction of accepted offers is $76 \%$, which shows a high level of successful trades. ${ }^{36}$ Some offers might be rejected, because subjects value fairness differently and the preference for fairness as captured by $\theta$ is private information, something we do not incorporate in our model. That accepted offers have, on average, higher prices lends support to this explanation.

We now turn to the ability of the subjects to exploit the gains from trade. Because the terminal value of a widget for a responder is $\$ 0.1$, while it is twice as large, $\$ 0.2$, for a proposer, each widget exchanged in a match generates earnings within a match of $\$ 0.1$. Given that the maximum number of widgets that can be exchanged is $\bar{\omega}=100$, the maximum earnings (ME) from trading in a match are $\$ 10$. We will compare the average earnings observed in our experiments with the ME, bearing in mind that a trade can be Pareto-efficient even when earnings are less than the ME. We will also describe how the average earnings are divided between the proposer and the responder. The earnings of a responder are $\$ 0.1(n-\omega)$ and the earnings of a proposer are $\$ 0.1(2 \omega-n)$, and so the total earnings are $\$ 0.1 \omega$. Accordingly, the earnings share of a proposer is $2-n / \omega$ and the earnings share of a responder is, $n / \omega-1$, where $n / \omega$ is the price of widgets in terms of notes.

RESULT \#3 (Earnings under perfect information). Under perfect information the subjects receive on average $58.6 \%$ of the maximum earnings of $\$ 10$. The average earnings share of responders is $19 \%$.

This surplus is split unequally: The average earnings for proposers and responders are $\$ 4.74$ and $\$ 1.12$, respectively. Conditional on trading, the average match realizes $78.9 \%$ of the ME. Finally, the average

\footnotetext{
${ }^{36}$ As a comparison, Duffy and Puzzello (2014), who implement the Lagos-Wright model in the lab, obtain acceptance rates in bilateral matches between $40 \%$ and $50 \%$. We attribute our relatively high acceptance rates to the following reasons. First, we chose linear payoffs (instead of strictly concave ones) that are easily computed, thereby minimizing the risk that subjects miscalculate incentive-compatible offers. Second, we do not have an additional stage where subjects have to choose their holdings of liquid assets (as in the original Lagos-Wright model). When we introduce such a stage (not in the paper), the acceptance rate falls to $64 \%$. Third, subjects do not have to form beliefs about the redemption value of their notes, as they would have to do in an environment with fiat money.
} 
earnings share of responders is $19 \%$. Altogether, the average earnings are below maximum earnings for two reasons: First, a quarter of all offers are rejected; second, conditional on a trade, about $20 \%$ of all widgets remain in the responders' hands.

Summary of Perfect Information (SI). Results 1-3 suggest that the behavior of the subjects is largely consistent with Hypothesis 1: Most offers are accepted and most trades are Pareto-efficient. The outcome differs from the SPE outcome according to which the entire match surplus is captured by the proposer. Furthermore, some offers with a positive surplus are rejected. Both observations are consistent with the notion that subjects value fairness, as typically observed in ultimatum games (Güth and Kocher, 2013).

\subsection{Moral hazard (MH)}

Table 2 summarizes key statistics from our MH sessions. For the discussion that follows, it is useful to distinguish between sessions with low costs of counterfeiting, $C \leq 4$, and sessions with high costs of counterfeiting, $C \geq 6$, as sessions in each group share similar characteristics. Recall that if $C=10$, then acquiring blue notes is a weakly dominant strategy, because blue and red notes are equally costly, but blue notes have a higher redemption value than red ones. Moreover, it is strictly optimal for the proposer to acquire blue notes if he believes that there is a positive probability that his offer will be rejected. In the opposite case where $C=0$, acquiring red notes is a weakly dominant strategy, and it is strictly optimal if the proposer believes that there is a positive probability that his offer will be accepted.

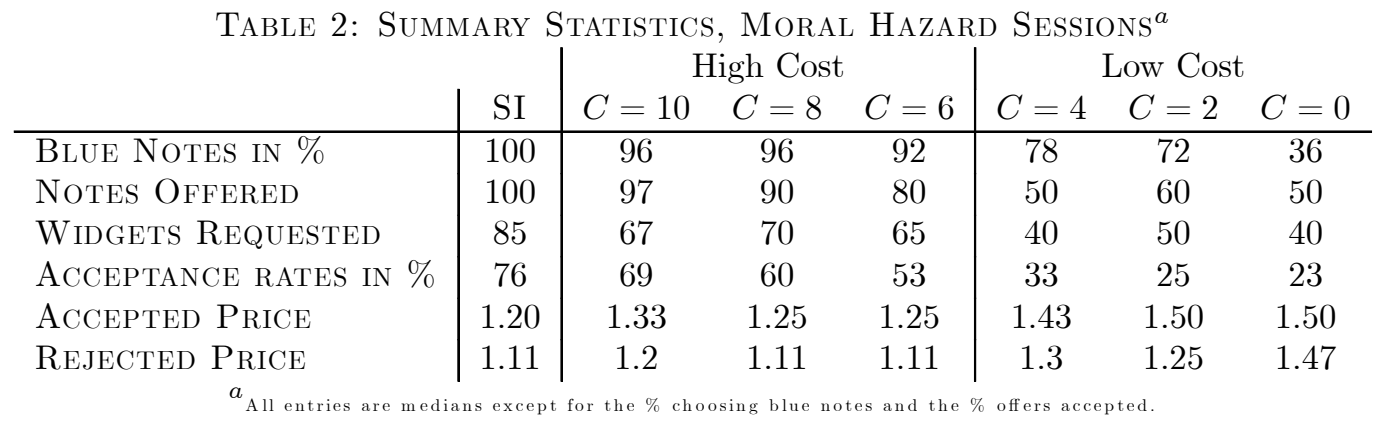

The following result provides stylized facts for offers both in terms of quantities (note offer, $n$ ) and prices (notes per widget, $n / \omega$ ).

RESULT \#4 (Offers under the threat of fraud). The median number of notes offered is lower under 
the threat of fraud than under perfect information $(n<100$ for all $C)$, and it is higher for a high cost of fraud $(C \geq 6)$ than a low cost of fraud $(C \leq 4)$. Median prices are higher for a low cost of fraud than a high cost.

The cost of fraud affects the quantities traded $(n$ and $\omega)$ and prices $(n / \omega)$ in accordance with theory. The median note offer and the median widget request are lower in the low-cost sessions than in the high-cost sessions. The median note offer decreases non-monotonically from $n=97(C=10)$ to $n=50(C=0)$, and the median widget request also decreases non-monotonically from $\omega=67(C=10)$ to $\omega=40(C=0)$.

For $C=10$, the median note offer, $n=97$, is close to its complete-information level, $n=100$. The median widget request, however, is lower, $\omega=67$ instead of $\omega=85$, suggesting that prices are higher to compensate sellers for the residual uncertainty about the quality of notes. In contrast, when fraud is costless, $C=0$, the median note offer and the median widget request plummet to $n=36$ and $\omega=50$. Thus, the possibility of fraud has a dramatic effect on the intensive margin of trade when fraud is costless.

In theory (see Hypothesis 2), the optimal note offer should be between $10 C$ and 100. If the offer is $10 C$, then the offer is accepted with certainty, and if the offer is strictly larger than $10 C$, the offer is accepted with some probability less than one. The acceptance probabilities are such that all offers are payoff equivalent, from the proposer's viewpoint. Figure 6 plots the theoretical boundaries for the offers - the red line (with squares) is the lower bound, $10 C$, while the green line (with triangles) is the upper bound, 100 - and the medians from the experiments (the blue line). The medians of the note offers are located between the theoretical boundaries for $C=0,2,4,6,8$.

The median (accepted) price is higher in low-cost sessions $(n / \omega \geq 1.43)$ than in high-cost sessions $(n / \omega \leq 1.33)$. This pattern also holds for rejected offers and if we separate blue-note and red-note offers. However, prices are barely affected within high-cost sessions and low-cost sessions. Furthermore, the median price of accepted offers is higher than the median price of rejected offers across all sessions. This finding is consistent with the SI session where responders require a minimum expected surplus in order to accept an offer. The minimum acceptable price to the responder in high-cost sessions is close to the one under perfect information (between 1.11 and 1.2). This reservation price increases significantly in low-cost sessions. 


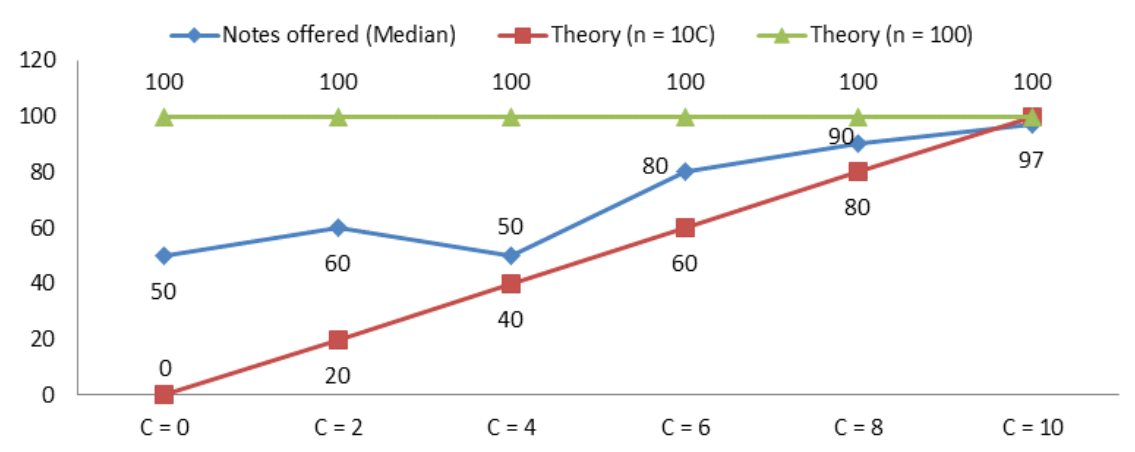

Figure 6: Median notes offered, MH Sessions

To formally test whether offers change as the cost of fraud changes, we conduct a nonparametric statistical analysis. Standard tests of differences in offers across treatments are problematic, because the offers are bounded with many offers at the boundary of $n=100$. Typical distributional assumptions will thus not be appropriate. We instead conduct a nonparametric bootstrapping procedure to test differences across treatments. From the set of $m$ experimental observations for a session, we draw with replacement a new sample of $m$ observations. ${ }^{37}$ We then calculate the statistic of interest (e.g., mean) for this new sample, and repeat the procedure 10,000 times for each session to obtain a pool of statistics (e.g., 10,000 means). After repeating this procedure for each session, we take the first statistic from two different sessions (e.g., $\mathrm{MH}_{8}$ and $\mathrm{MH}_{2}$ ), calculate the difference between the two statistics, and then repeat for the rest of the statistics from each session to construct a distribution of differences with which to make statistical inferences.

\footnotetext{
${ }^{37}$ For most variables of interest (price, acceptance rate, blue notes rate, etc.), all observations are used. Because price can be undefined if 0 widgets are requested, we only use those observations in which the widgets requested is not 0.
} 
Mean notes offered

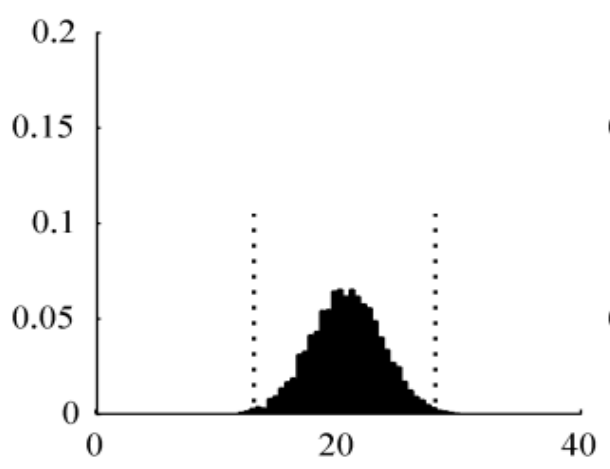

Mean price

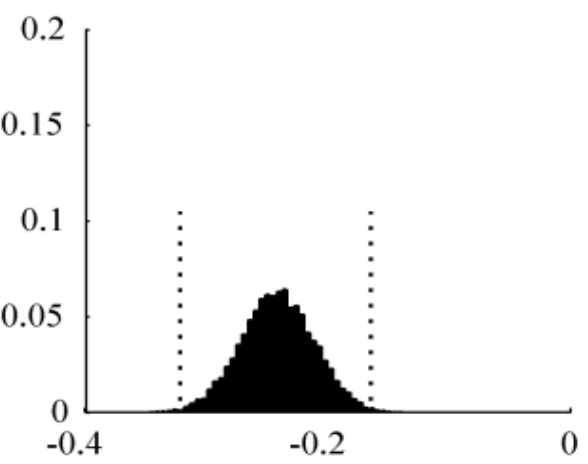

Figure 7: Nonparametric difference distributions, $\mathrm{MH}_{10}-\mathrm{MH}_{0}$

Table 3: 99\% Confidence Intervals for Difference in Means, Si and MH Sessions

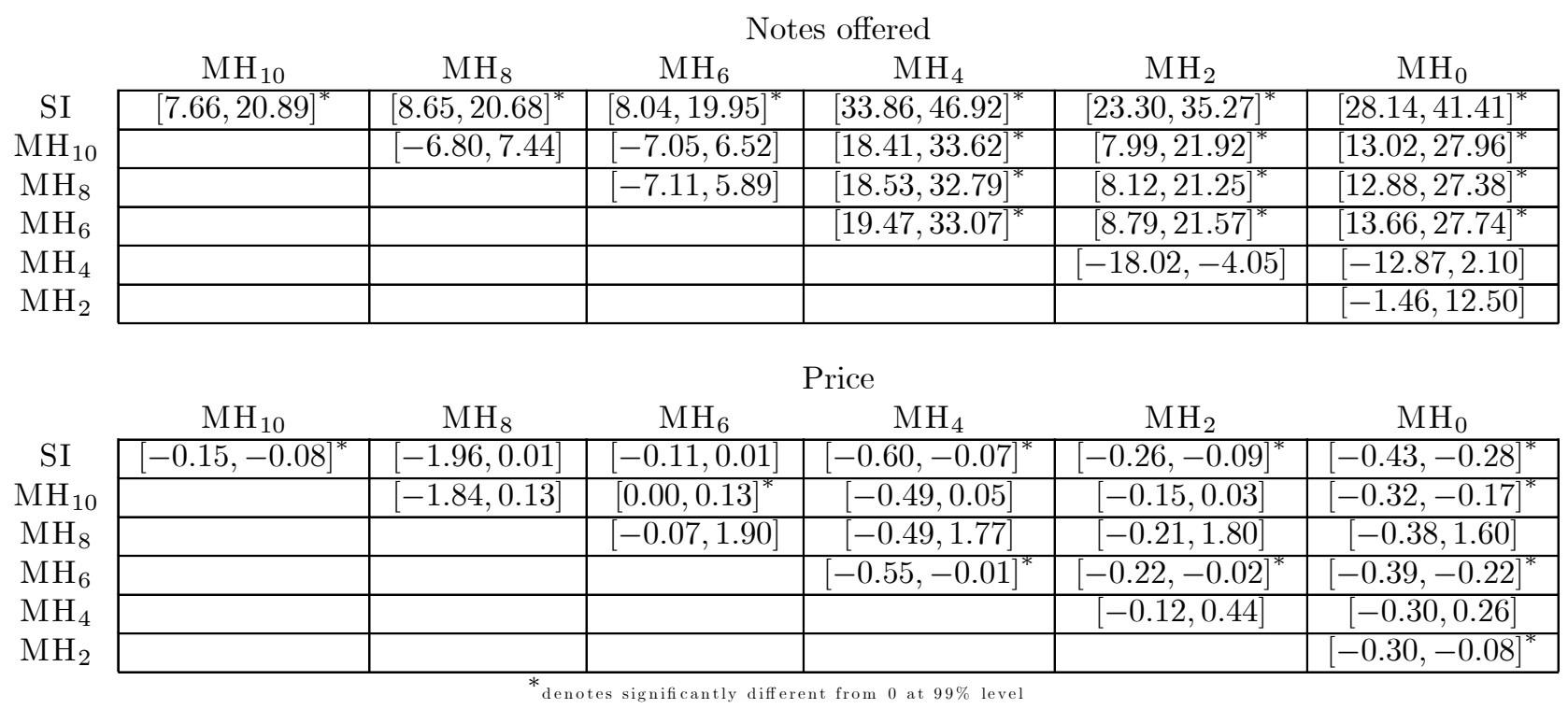

Figure 7 displays the difference distributions for $\mathrm{MH}_{10}-\mathrm{MH}_{0}$ for notes offered and price. These two sessions represent the starkest difference in cost for the $\mathrm{MH}$ sessions. The $99 \%$ confidence intervals marked by dotted lines reveal that the difference in mean note offers between $\mathrm{MH}_{10}$ and $\mathrm{MH}_{0}$ is positive with high statistical significance, and the difference in price is negative with high statistical significance. Table 3 provides the nonparametric confidence intervals for mean note offers and mean price for all SI and MH session comparisons. For mean note offers, we do not always find statistical differences when comparing sessions 
with small differences in $C$, but we typically find statistical differences for sessions with large differences in $C$. Observed prices exhibit greater variance, yet a similar pattern is found: The mean prices are statistically different across sessions with large differences in $C$. Overall, the nonparametric analysis provides formal statistical evidence that offers sizes decrease and prices increase in response to a growing threat of fraud. This result has implications for policy analysis as it shows that any measure that raises the cost of producing fraudulent means of payment has the potential to increase the volume of trade.

RESULT \#5 (Acceptance rates under the threat of fraud). Fewer offers are accepted under the threat of fraud than under perfect information. Moreover, the share of accepted offers falls monotonically from $69 \%(C=10)$ to $23 \%(C=0)$.

We first discuss the case when fraud is costless. For $C=0$, a key prediction of Hypothesis 2 is that when the cost of producing fraudulent (red) notes approaches zero, notes become illiquid and no longer serve as a means of payment. In accordance with this prediction, when notes can be counterfeited at no cost in session $\mathrm{MH}_{0}$, about $75 \%$ of offers are rejected. This result provides a stark example of asset illiquidity generated by the threat of fraudulent practices. It is still surprising that there is trade taking place, given that producing fraudulent notes is a weakly dominant strategy. This observation can be rationalized by the fact that $36 \%$ of offers involved blue notes. Why some proposers acquired blue notes if they expected responders to accept offers is unclear but may be due to preferences concerning non-monetary considerations by the subject, such as honesty or fairness, that are not commonly known.

We next discuss the intermediate cases $C=2,4,6,8$. In theory (see Hypothesis 2), if $n \leq 10 C$, then the offer is accepted with certainty, $p=1$, whereas if $n>10 C$, then the offer is accepted with probability $p=10 C / n<1$. As shown above, the median note offer is typically above $10 C$, and hence in theory it should only be partially accepted. If we compute the theoretical acceptance rate for the median offer, $p^{\text {theory }}=10 C / n$, we find $p^{\text {theory }}=1 / 3,4 / 5,3 / 4,8 / 9$ for $C=2,4,6,8$. See Table 4 . These theoretical acceptance rates tend to increase with the cost of fraud, in accordance with the experimental evidence. However, theory over-predicts the acceptance rate, which was also the case under perfect information. Our nonparametric analysis (see the supplement appendix) generally finds the decrease in the acceptance rate as $C$ drops to be statistically significant. 
The previous results show that subjects do trade under the threat of fraud. For all $C>0$, there are least $25 \%$ of all offers that are accepted and the median number of notes offered does not fall below 50 . The fact that trade is resilient to the threat of fraud is in contradiction with the equilibrium concept used in Nosal and Wallace (2007) according to which trades should shut down but it is consistent with our equilibrium notion.

Table 4: Comparison with Theory ${ }^{a}$

\begin{tabular}{l|ccc|ccc} 
& $C=10$ & $C=8$ & $C=6$ & $C=4$ & $C=2$ & $C=0$ \\
\hline MEDIAN $n$ & 97 & 90 & 80 & 50 & 60 & 50 \\
MEDIAN $\omega$ & 67 & 70 & 65 & 40 & 50 & 40 \\
$p^{\text {data }}$ IN \% & 69 & 60 & 53 & 33 & 25 & 23 \\
$p^{\text {theory }}$ IN \% & 100 & 89 & 75 & 80 & 33 & 0 \\
$a_{\text {All entries are medians except for the acceptance rate. }}$
\end{tabular}

Finally, we discuss the case when fake notes cost the same as genuine notes, $C=10$. Theory predicts that offers should be attributed to blue-note holders and hence should be accepted with certainty. In our experiment, only $69 \%$ of all offers are accepted, which is less than the $76 \%$ under perfect information. This could be explained by the fact that $4 \%$ of proposers chose red notes with the result that some subjects received red notes, which might have made them more cautious in their acceptance strategy.

RESULT \#6 (The extent of fraud). When fraud is costless, $C=0,64 \%$ of offers involve fraudulent notes. When fraud is costly, $C \geq 2$, more than $72 \%$ of offers involve genuine notes, and when $C \geq 6$, more than $92 \%$ of offers involve genuine notes.

A stark prediction of the theory (Hypothesis 2) is that no fraud takes place in equilibrium if fraud is costly, $C>0$. Intuitively, if fraud is correctly anticipated, then it is not beneficial to the proposer and hence it should not happen. In the lab, as in actual economies, some fraud takes place. ${ }^{38}$ However, in high-cost sessions, the fraction of fraudulent notes is less than $8 \%$, which we view as remarkably consistent with a no-fraud equilibrium. Even for low costs of fraud, $C=2,4$, the share of blue-note offers is large ( $72 \%$ and $78 \%)$. Moreover, fraud is monotonically increasing as the cost of counterfeiting decreases from $4 \%(C=10)$

\footnotetext{
${ }^{38}$ The no-fraud proposition is also at odds with real-world evidence. Counterfeiting has been documented for all major currencies. In 2005 in the U.S., $\$ 61$ million of counterfeit currency was passed on to the public, 3,717 counterfeiters were arrested, and 611 counterfeiting plants were suppressed. See United States Treasury Department (2006). As documented by Mihm (2007), counterfeiting was a widespread phenomenon in the U.S. during the 19th century.
} 
to $64 \%(C=0)$, and this decline is statistically significant. So, while broadly consistent with the theory, the experimental evidence is more nuanced than the sharp no-counterfeiting result.

RESULT \#7 (Note retention as a signaling mechanism). Blue-note offers tend to involve fewer notes and a lower price than red-note offers, and they tend to be accepted more often.
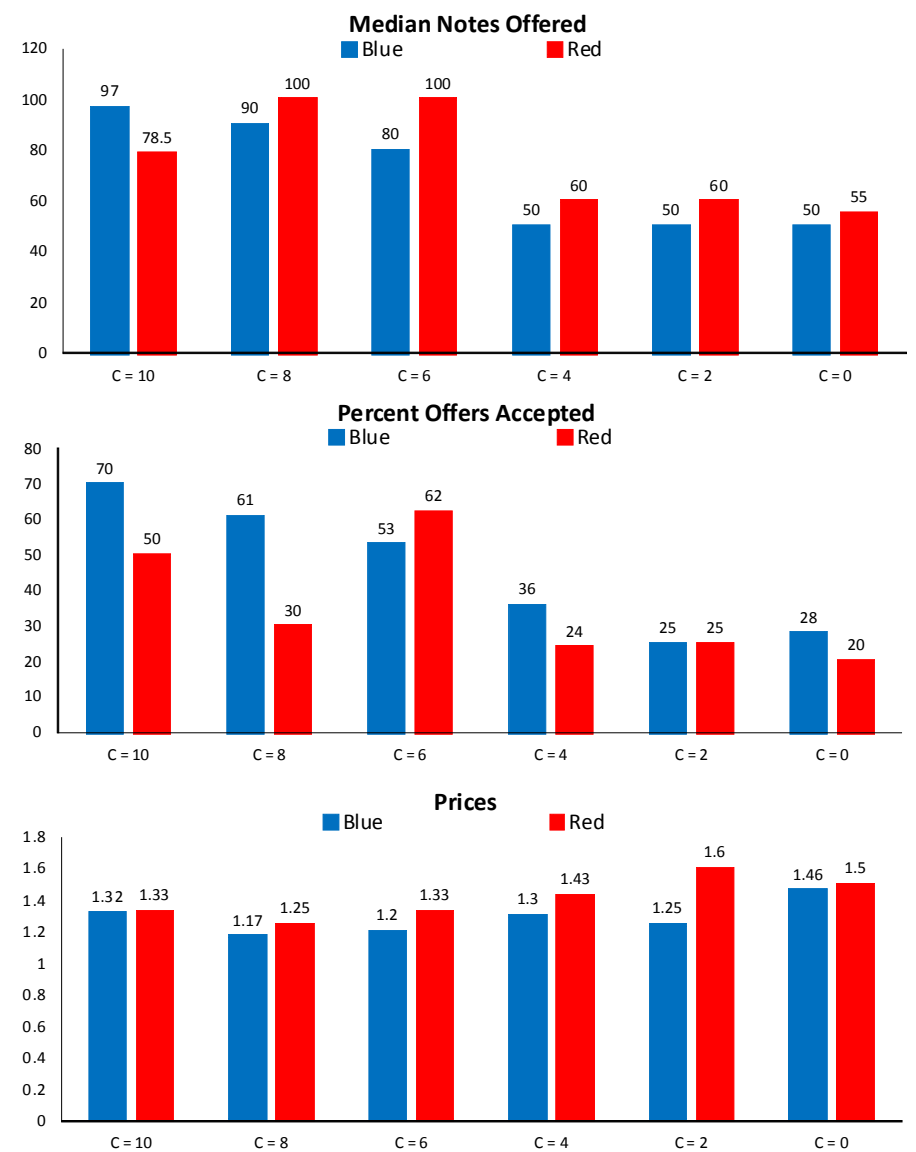

Figure 8: Offered notes, acceptance, and prices by type of notes, MH sessions

The top panel of Figure 8 compares offers made by proposers with blue notes and proposers with red notes. Except for $C=10$, where the fraction of red-note holders is only $4 \%$, blue-note proposers consistently offer fewer notes than red-note proposers. This finding is consistent with the result from the adverse-selection literature according to which individuals can signal the quality of their assets, and hence obtain better terms 
of trade, by retaining a fraction of those assets (see, e.g., De Marzo and Duffie, 1999, and Rocheteau, 2011). It is also consistent with a Gresham's Law outcome according to which bad monies (red notes) drive good monies (blue notes) out (see, e.g., Velde, Weber, and Wright, 1999).

As the cost of counterfeiting decreases from $C=10$ to $C=0$, the acceptance rate for blue notes decreases from $97 \%$ to $28 \%$, while the acceptance rate of red notes decreases from $50 \%$ to $20 \%$. As shown in the middle panel of Figure 8, blue-note holders seem to successfully signal the quality of their notes as the acceptance rates for blue notes are higher or equal to those for red notes in five out of six $\mathrm{MH}$ experiments (the exception is the session with $C=6$ ).

The bottom panel of Figure 8 shows that the price offered by red-note holders is always higher than the price offered by blue-note holders. This is again consistent with a signaling mechanism according to which the individual with high-quality assets retains some of his assets but asks for a better price. Alternatively, it might be that red-note holders were simply attempting to induce responders to accept their proposals by offering higher prices for widgets. If this was the case, these attempts were not successful, since, as shown above, blue-note offers were accepted with a higher probability than red-note offers.

RESULT \#8 (Earnings under the threat of fraud). In high-cost sessions, $C \geq 6$, the average earnings are above $28 \%$ of the maximum earnings of $\$ 10$. In low-cost sessions, $C \leq 4$, they are below $8 \%$.

The high-cost sessions feature a monotonic decrease in subjects' earnings, from $\$ 4(C=10)$, to $\$ 3.5$ $(C=8)$ and $\$ 2.8(C=6) .{ }^{39}$ These earnings are smaller than the ones obtained under perfect information (SI), \$5.9, suggesting that the threat of fraud impairs subjects' ability to exploit gains from trade. The earnings are even lower, and slightly increasing, in the low-cost sessions. ${ }^{40}$ The average match exploits more than $28 \%$ of the ME in high-cost sessions (more than $57 \%$ conditional on trading) and less than $8 \%$ in low-cost sessions (less than $37 \%$ conditional on trading).

Figure 9 displays the average earnings as a percentage of the ME. The difference between the earnings conditional on trading and the unconditional earnings is positive by definition because rejected offers generate

\footnotetext{
${ }^{39}$ With moral hazard, the maximum earnings (ME) are $\$ 10$ as in the perfect information (SI) session. A trade generates $\$ 10$ for the match if all widgets are exchanged and if $C>0$, no money is wasted on red notes.

${ }^{40}$ The average earnings discussed below are the mean earnings of the subjects across all rounds. They do not coincide with the payout of the experiments, since the payouts are based on one round chosen at random.
} 


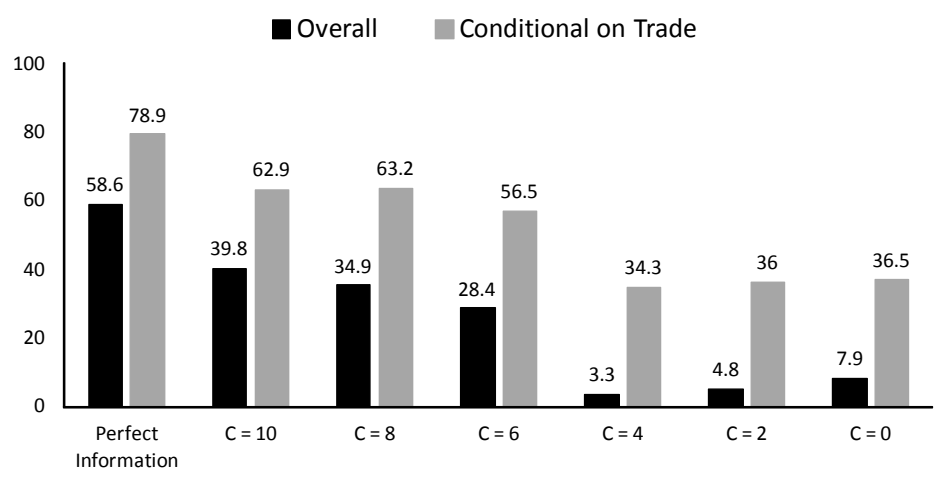

Figure 9: Average earnings as percentage of ME, SI and MH sessions

no earnings. The size of the difference reflects the contribution of the extensive margin (the acceptance rate) to the overall earnings. As the figure suggests, the extensive margin plays a crucial role for low costs of fraud.

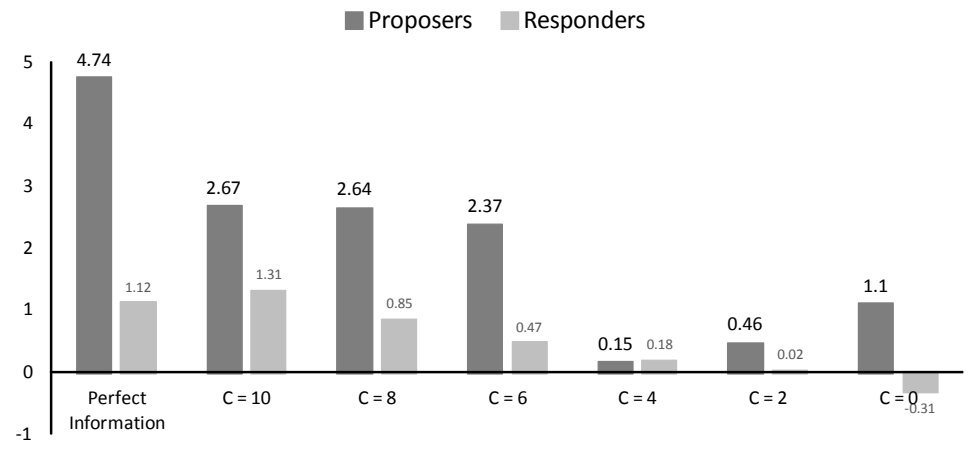

Figure 10: Average earnings by role, SI and MH sessions

Earnings can be separated by player role, as shown in Figure 10. Proposers' average earnings are larger than $\$ 2.4$ in high-cost sessions and lower than $\$ 1.1$ in low-cost sessions. Responders' average earnings are higher than $\$ 0.5$ in high-cost sessions and lower than $\$ 0.2$ in low-cost sessions. When the moral hazard problem was very severe, $C=0$, responders made an average loss of $\$ 0.3$.

RESULT \#9 (Earning shares under moral hazard). Proposers' average earnings share is constant for high-cost sessions and increasing in $C$ for low-cost sessions. Responders' earnings share is positive, 


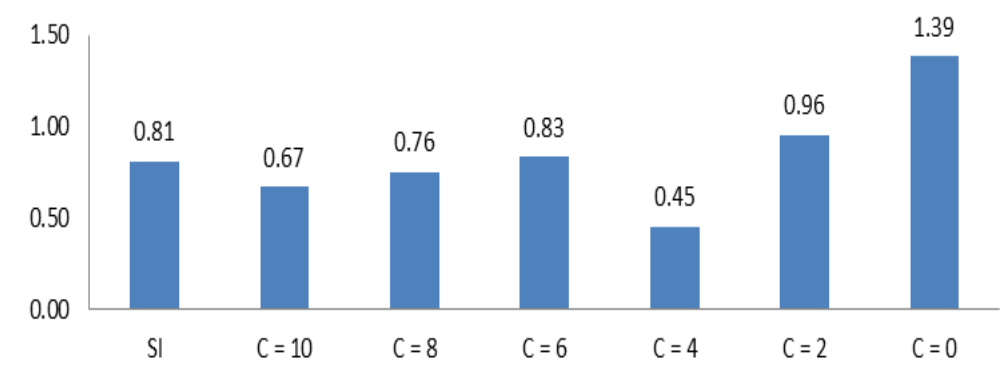

Figure 11: Average fraction of surplus for proposers, SI and MH sessions

except for $C=0$.

The average fraction of earnings obtained by proposers is shown in Figure 11. In high-cost sessions, the fraction of total earnings obtained by proposers is roughly constant and equal to the perfect-information (SI) share, being about $80 \%$. In low-cost sessions, the responder share is increasing in $C$ and is larger than $100 \%$ when fraud is costless (see Figure 11).

Summary of moral hazard (MH). Results 4-8 suggest that the behavior of the subjects is largely consistent with Hypothesis 2. If fraud is costless, the threat of fraud strongly reduces the subjects' ability to exploit the gains from trade since very few offers are accepted and the size of accepted trades are small. If fraud is costly, a vast majority of offers involve genuine notes and the fraction of accepted offers, trade sizes, and earnings increase with the cost of fraud.

\subsection{Testing the reverse-ordered game refinement}

The theory analyzes the OTC bargaining game under the threat of fraud by adopting the reverse-ordered game refinement for signaling games with endogenous types of In and Wright (2011). ${ }^{41}$ In the following, we test whether the reverse-ordered game generates similar outcomes as the original bargaining game by letting subjects play the reverse-ordered game in the lab. Figure 12 illustrates key similarities between the MH and RMH sessions that we discuss here.

\footnotetext{
${ }^{41}$ Recall that according to this refinement, the outcome of the original bargaining game can be predicted from the subgame perfect equilibrium of a strategically equivalent game. In that game, the proposer chooses first his observed action (the offer) and then he chooses the unobserved action (whether to acquire blue or red notes).
} 

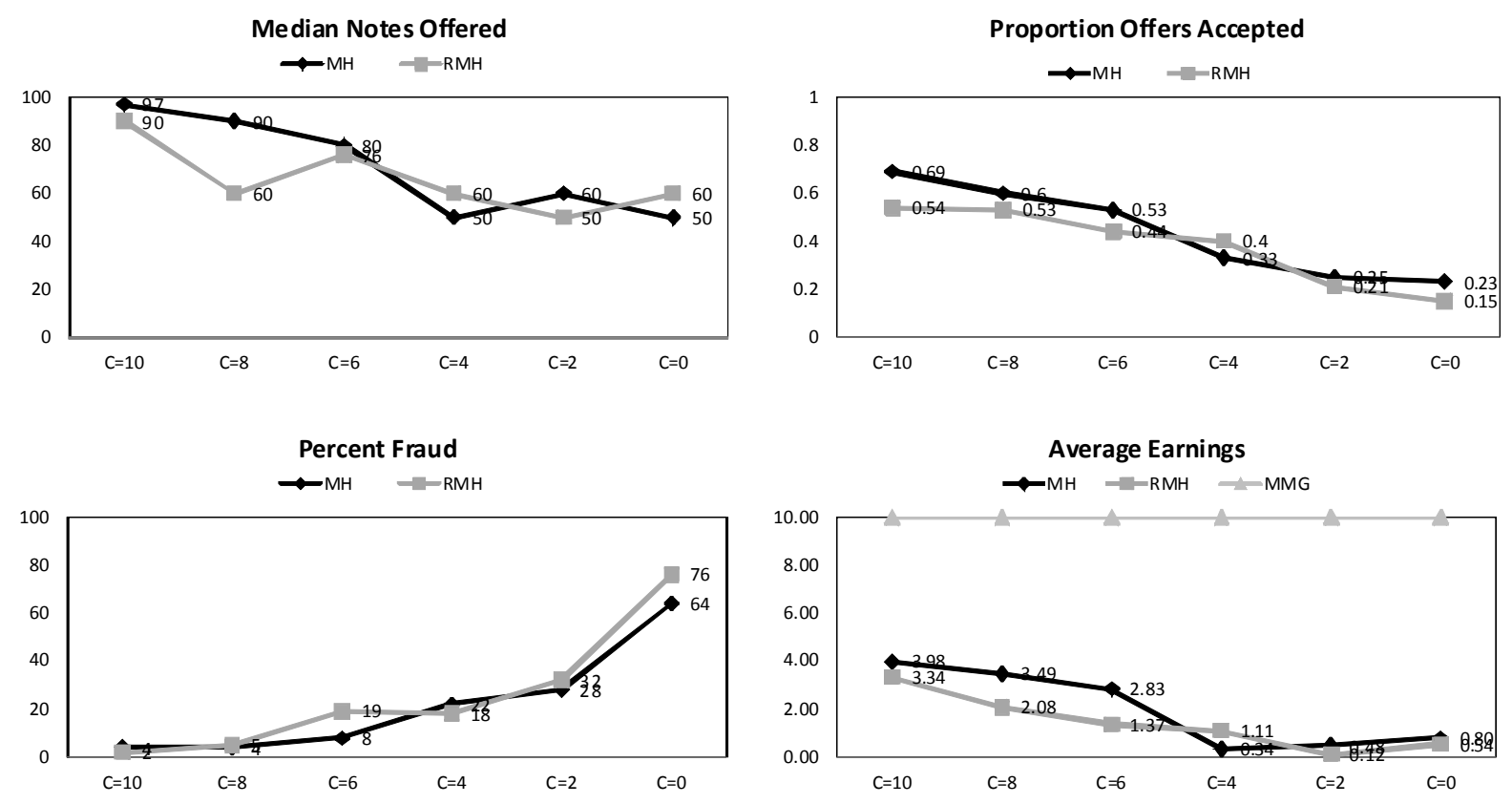

Figure 12: MH and RMH Sessions Compared

RESULT \#10 (Accuracy of the reverse-ordered game refinement) Outcomes of the reversed-ordered games in terms of offers, acceptability, fraud, and gains from trade, are largely consistent with those of the original game.

In accordance with Result 4, note offers are smaller than in the perfect information case, $n<100$, and they decrease as fraud becomes cheaper. Though notes offered are statistically different with $C=2,4,6,8$ according to our nonparametric statistical analysis (see supplement appendix), they follow the same general pattern as $C$ changes, as seen in Figure 12. The primary exception is $C=8$ where $n$ is much smaller in the reverse-ordered game.

In accordance with Result 5, acceptance rates are smaller under the threat of fraud than under perfect information. Relative to the original bargaining game, acceptance rates are lower but of similar magnitude. In the $\mathrm{RMH}$ session, the acceptance rate picks at $54 \%$ relative to $69 \%$ in the $\mathrm{MH}$ sessions, and it falls down to $15 \%$ relative to $23 \%$ in the $\mathrm{MH}$ sessions. We also find that the acceptance rate is monotonically increasing in $C$ in both versions of the game. We find no statistical difference in acceptance rates across the $\mathrm{MH}$ and 
$\mathrm{RMH}$ sessions, the sole exception being when $C=10$.

Figure 12 also shows that the fraud levels are decreasing in $C$ and of equal magnitude in both $\mathrm{RMH}$ and $\mathrm{MH}$ sessions. When $C=10$, the share of fraud is only $2 \%$ in the reverse-ordered, and it remains less than $5 \%$ when $C=8$. The amount of fraud is higher, $19 \%$, for a medium-high cost of fraud. Still, the share of fraudulent offers is less than one third for all $C>0$, which is consistent with Result 5 . When fraud is costless, $C=0$, the fraud share jumps to $76 \%$, which is also consistent with the theory. There is no statistically significant difference for all $C$ except $C=6$.

Finally, we also find that mean earnings are increasing in $C$ in both $\mathrm{RMH}$ and $\mathrm{MH}$ sessions. Except for $C=4$ earnings are higher when subjects play the original game. But the main message remains that subjects experience difficulties to exploit the gains from trade when the bargaining game is subject to the threat of fraud.

Summary of reverse moral hazard (RMH). The reverse-ordered OTC bargaining game generates similar, often statistically identical, subject behavior in terms of offers, acceptability, and fraud compared to the original bargaining game. Hence, it is a reasonable refinement for OTC bargaining games with hidden actions. Furthermore, the fact that we observe similar behavior across slightly different OTC bargaining games provides additional support for the robustness of our experimental results.

\subsection{Further interpretation: Hidden action vs adverse selection}

In order to better understand the effects of informational frictions on trade, we ran two additional sessions where proposers are privately informed about the terminal value of their notes, but are not able to choose the value; i.e., Nature rather than the proposer chooses the value of the notes. ${ }^{42}$ We label these new sessions $\mathrm{AS}_{0.9}$ and $\mathrm{AS}_{0.7}$, where $\mathrm{AS}$ stands for adverse selection and the index indicates the probability of blue notes, $\pi \in\{0.9,0.7\}$. Some key statistics from $\mathrm{AS}_{0.9}$ and $\mathrm{AS}_{0.7}$ are compared with $\mathrm{MH}$ sessions in Table 4.

We first compare the MH session with $C=6$ with the AS session with $\pi=0.90$. When $C=6$ the fraction of proposers who opted for blue notes is $92 \%$, which is comparable to the fraction in the AS experiment (where the realized fraction was $88 \%$ ). The median note offer and the median widget offer are slightly lower

\footnotetext{
${ }^{42}$ In all these sessions, the subjects had access to an on-screen calculator to help them compute payoffs for different hypothetical offers.
} 
in the MH session than in the AS session, $92>88$ and $80>85$, but the acceptability of offers is slightly higher in the $\mathrm{MH}$ session, $53 \%>46 \%$. Overall, the total number of notes that change hands is similar across the two treatments. The median offered price in the MH session, 1.25, is slightly lower than the one in the AS session, 1.29. Finally, the earnings in the MH session, $\$ 2.84$, are slightly higher than the one in the AS session, \$2.62. This comparison suggests that whether the private information problem is generated by subjects' hidden actions or by Nature is not too relevant for the outcomes of the OTC bargaining games, at least if the amount of fraud is small.

Table 4: Comparison Between MH and $\mathrm{AS}^{a}$

\begin{tabular}{l|cc|cc} 
& $C=6$ & $\mathrm{AS}_{0.9}$ & $C=2$ & $\mathrm{AS}_{0.7}$ \\
\hline BluE NOTES IN \% & 92 & 88 & 72 & 79 \\
NOTES OFFERED & 80 & 85 & 60 & 60 \\
WIDGETS REQUESTED & 65 & 67 & 50 & 50 \\
PRICE OFFERED & 1.25 & 1.29 & 1.33 & 1.29 \\
ACCEPTANCE RATES IN \% & 53 & 46 & 25 & 39 \\
EARNINGS & 2.84 & 2.62 & 0.5 & 1.65 \\
$a_{\text {all entries are medians except for the acceptance rate and earnings (average in \$). }}$
\end{tabular}

Next, we compare the MH session when $C=2$ that has an endogenous fraction of blue notes of $72 \%$ with AS session when $\pi=70 \%$ (in the experiment the realized $\pi$ is $79 \%$ ). In each case, the median number of notes offered is 60 and the median number of widgets requested is 50. So the intensive margin of the trade is identical. However, the acceptance rate of offers is significantly lower in the $\mathrm{MH}$ session, $25 \%$, compared to $39 \%$ in the AS session. The threat of fraud, therefore, appears to have a larger effect on overall trade, since responders tend to "trust" Nature more than other subjects. As a result, the average earnings are much smaller in the MH session, $\$ 0.5$, compared to the AS session, $\$ 1.65$. Some of this difference is due to the fact that about $30 \%$ of subjects bought red notes at the cost of $\$ 2$, which reduces the average gain by about \$0.6. The rest of the difference comes from the lower acceptance rate.

\section{Conclusion}

We have presented a simple theory of bargaining games in decentralized markets under perfect and private information. In these two-dimensional bargaining games, agents trade a commodity (called 'widget') that they value differently — hence the presence of gains from trade - for a medium of exchange (called 'note') that has the same value for all market participants. In order to assess the effects of informational frictions 
regarding the common value of the medium of exchange, we compared a benchmark case where agents are symmetrically informed (SI) about the terminal value of notes with six conditions in which note-holders can take hidden actions $(\mathrm{MH})$ to affect the terminal value of the notes. After having derived a set of theoretical predictions for this environment under different information structures, we took the model to a laboratory setting to test its predictions.

The main lessons of this exercise are as follows. First, the experimental subjects' behavior in the bargaining game under perfect information is largely consistent with the theory: Most offers are individually rational and close to the Pareto frontier, and a large fraction of offers are accepted. Second, the experimental data is also consistent with the predictions of the model under the threat of fraud with noteworthy differences. We found that the possibility of fraud affects trade on both the intensive and extensive margins: Subjects trade less often, and when offers are accepted they trade smaller quantities. As a result, the threat of fraud strongly reduces the ability of subjects to achieve the gains from trade. A policy implication of this result is that measures to improve the recognizability of means of payment - such as the periodic redesign of currencies by central banks - have real effects by increasing the volume of trade and are welfare improving. While the theory predicts that no fraud should occur in equilibrium (provided that fraud is costly), we observed some fraud in the experiments. However, the amount of fraud was always small. Because the volume of fraud is negatively correlated with the cost of fraud, it provides an indicator of the recognizability of the means of payment. When fraud was costless, the volume of trade was very low but positive, in contrast to the complete shut-down predicted by theory. So, even though trade is affected by the threat of fraud, it is also resilient. Third, behavior in the reverse-ordered game closely matches the behavior in the original ordered setting, suggesting that the reverse-ordered game is a valuable refinement for the study of OTC bargaining games under hidden actions.

Our model is simple and tractable and can be extended in many ways. For instance, it can be used to study how incentives to acquire information matter for asset and market liquidity. One can also use our model to investigate other dimensions of collateralized trades, such as haircuts, and how these dimensions are affected by various informational frictions. We leave these different questions for future research. 


\section{References}

[1] Arrow, Kenneth (1971). Essays in the Theory of Risk-Bearing. Chicago, IL: Markham Publishing Company.

[2] Aruoba, S. Boragan, Guillaume Rocheteau and Christopher Waller (2007). "Bargaining and the value of money," Journal of Monetary Economics 54, 2636-2655.

[3] Banarjee, Abhijit and Eric Maskin (1996). "A Walrasian theory of money and barter," Quarterly Journal of Economics 111, 955-1005.

[4] Barnett, Harold (2012). "And some with a fountain pen: Mortgage fraud, securitization and the subprime bubble." in How They Got Away with it: White-Collar Crime and the Financial Meltdown, edited by Susan Will, Stephen Handelman and David C. Brotherton. New York: Columbia University Press.

[5] Berentsen, Aleksander and Guillaume Rocheteau (2002). "On the efficiency of monetary exchange: How divisibility of money matters," Journal of Monetary Economics 49, 1621-1649.

[6] Berentsen, Aleksander and Guillaume Rocheteau (2003). "Money and the gains from trade," International Economic Review 44, 263-297.

[7] Berentsen, Aleksander and Guillaume Rocheteau (2004). "Money and information," Review of Economic Studies 71, 915-944.

[8] Bolton, Gary, and Axel Ockenfels (2000). "ERC: A theory of equity, reciprocity, and competition," The American Economic Review 90, 166-193.

[9] Burdett, Kenneth, Alberto Trejos, and Randall Wright (2001). "Cigarette money," Journal of Economic Theory 99, 117-142.

[10] Brown, Paul (1996). "Experimental evidence on money as a medium of exchange," Journal of Economic Dynamics and Control 20, 583-600.

[11] Camargo, Braz and Benjamin Lester (2013). "Subsidizing price discovery," Research Department, Federal Reserve Bank of Philadelphia, Working paper No. 13-20.

[12] Camera, Gabriele, and Marco Casari (2014). "The coordination value of monetary exchange: Experimental evidence." American Economic Journal: Microeconomics 6, 290-314.

[13] Camera, Gabriele, Marco Casari, and Maria Bigoni (2003). "Money and trust among strangers," Proceedings of the National Academy of Sciences of the United States of America 110, 14889-14893.

[14] Camera, Gabriele, Marco Casari, and Stefania Bortolotti (2014). "An experiment on retail payments systems," SAFE Working Paper No. 49.

[15] Camera, Gabriele, Charles Noussair, and Steven Tucker (2003). "Rate-of-return dominance and efficiency in an experimental economy," Economic Theory 22, 629-660.

[16] Camerer, Colin F. (2003). Behavioral Game Theory: Experiments in Strategic Interaction. Princeton University Press.

[17] Cho, In-Koo and David Kreps (1987). "Signaling games and stable equilibria," Quarterly Journal of Economics 102, 179-221. 
[18] Cuadras-Morató, Xavier (1994). "Commodity money in the presence of goods of heterogenous quality," Economic Theory 4, 579-591.

[19] DeMarzo, Peter and Darrell Duffie (1999). "A liquidity-based model of security design," Econometrica 67, 65-99.

[20] Duffie, Darrell, Gârleanu, Nicolae, and Lasse Pedersen (2005). "Over-the-counter markets," Econometrica $73,1815-47$.

[21] Duffie, Darrell (2012). Dark Markets: Asset Pricing and Information Transmission in Over-the-Counter Markets. Princeton Lectures in Finance.

[22] Duffy, John (2008). "Macroeconomics: A survey of laboratory research," Working Papers 334, University of Pittsburgh, Department of Economics, revised March 2008.

[23] Duffy, John, and Jack Ochs. 1999. "Emergence of money as a medium of exchange: An experimental study." American Economic Review, 89(4): 847-877.

[24] Duffy, John and Jack Ochs (2002). "Intrinsically worthless objects as media of exchange: Experimental evidence," International Economic Review 43, 637-73.

[25] Duffy, John, and Daniela Puzzello (2014). "Gift exchange versus monetary exchange: Experimental evidence," American Economic Review 104, 1735-76.

[26] Engineer, Merwan and Shouyong Shi (1998). "Asymmetry, imperfectly transferable utility, and the role of fiat money in improving terms of trade," Journal of Monetary Economics 41, 153-183.

[27] Engineer, Merwan and Shouyong Shi (2001). "Bargains, barter, and money," Review of Economic Dynamics 4, 188-209.

[28] Fehr, Ernst, and Klaus Schmidt (1999). "A theory of fairness, competition, and cooperation," The Quarterly Journal of Economics 114, 817-868.

[29] Fischbacher, Urs (2007). "z-Tree: Toolbox for Ready-made Economic Experiments," Journal of Experimental Economics 10, 171-178.

[30] Forsythe, Robert, Lundholm, Russell, and Thomas Rietz (1999). "Cheap talk, fraud, and adverse selection in financial markets: some experimental evidence," Review of Financial Studies 12, 481-518.

[31] Forsythe, Robert, Kennan, John and Barry Sopher (1991). "An experimental analysis of strikes in bargaining games with one-sided private information," American Economic Review 81, 253-78, March.

[32] Gorton Gary B and Andrew Metrick (2010). "Haircuts," Review, Federal Reserve Bank of St. Louis, issue Nov., 507-520.

[33] Guerrieri, Veronica and Robert Shimer (2014). "Dynamic adverse selection: A theory of illiquidity, fire sales, and flight to quality," American Economic Review 104, 1875-1908.

[34] Güth, Werner and Martin Kocher (2013). "More than thirty years of ultimatum bargaining experiments: Motives, variations, and a survey of the recent literature," CESifo Working Paper Series 4380, CESifo Group Munich.

[35] Harrison, Glenn and Michael McKee (1985). "Experimental Evaluation of the Coase Theorem," Journal of Law and Economics 28, 653-670. 
[36] Hoffman, Elizabeth and Matthew Spitzer (1982). "The Coase Theorem: Some Experimental Tests," Journal of Law and Economics 25, 73-98.

[37] Hopenhayn, Hugo and Ingrid Werner (1996). "Information, liquidity, and asset trading in a random matching game," Journal of Economic Theory 68, 349-379.

[38] Hu, Tai-wei (2013) "Imperfect recognizability and coexistence of money and higher-return assets," Economic Theory 53, 111-138.

[39] Hu, Tai-wei, John Kennan, and Neil Wallace (2009). "Coalition-proof trade and the Friedman rule in the Lagos-Wright model," Journal of Political Economy 117, 116-137.

[40] In, Younghwan and Julian Wright (2011). "Signaling Private Choices," manuscript.

[41] Jacquet, Nicolas, and Serene Tan (2012). "Money and asset prices with uninsurable risks," Journal of Monetary Economics 59, 784-797.

[42] Jevons, William (1875). Money and the Mechanism of Exchange, London: C. Kegan Paul.

[43] Judson, Ruth, and Richard Porter (2003). "Estimating the worldwide volume of counterfeit U.S. currency: Data and extrapolation," Working Paper of the Division of Monetary Affairs, Board of Governors of the Federal Reserve System.

[44] Kagel, John, Kim, Chung and Donald Moser (1996). "Fairness in ultimatum games with asymmetric information and asymmetric payoffs," Games and Economic Behavior 13, 100-110.

[45] Kalai, Ehud (1977). "Proportional solutions to bargaining situations: Interpersonal utility comparisons," Econometrica 45, 1623-1630.

[46] Keys, Benjamin J., Tanmoy Mukherjee, Amit Seru, and Vikrant Vig (2010). "Did securitization lead to lax screening? Evidence from subprime loans," Quarterly Journal of Economics 125, 307-362.

[47] Kiyotaki, Nobuhiro and Randall Wright (1989). "On money as a medium of exchange," Journal of Political Economy 97, 927-954.

[48] Kohlberg, Elon and Jean-Francois Mertens (1986). "On the strategic stability of equilibria." Econometrica 54, 1003-37.

[49] Lagos, Ricardo and Guillaume Rocheteau (2009). "Liquidity in asset markets with search frictions," Econometrica 77, 403-426.

[50] Lagos, Ricardo and Randall Wright (2005). "A unified framework for monetary theory and policy analysis," Journal of Political Economy 113, 463-484.

[51] Lester, Benjamin, Postlewaite, Andrew and Randall Wright (2011). "Information and liquidity," Journal of Money, Credit and Banking, 43, 355-377.

[52] Li, Yiting (1995). "Commodity money under private information," Journal of Monetary Economics 36, 573-592.

[53] Li Yiting, and Guillaume Rocheteau (2011). "On the threat of counterfeiting," Macroeconomic Dynamics $15,10-41$. 
[54] Li Yiting, Guillaume Rocheteau and Pierre-Olivier Weill (2012). "Liquidity and the threat of fraudulent assets," Journal of Political Economy 120, 1223-1224.

[55] Mihm, Stephen (2007). A nation of counterfeiters: Capitalists, con men, and the making of the United States, Cambridge, Harvard University Press.

[56] Mitzkewitz, Michael and Rosmarie Nagel (1993). "Experimental results on ultimatum games with incomplete information," International Journal of Game Theory 22, 171-98.

[57] Nosal, Ed and Neil Wallace (2007). "A model of (the threat of) counterfeiting," Journal of Monetary Economics 54, 229-246.

[58] Rabin, Matthew (2000). "Risk aversion and expected-utility theory: A calibration theorem," Econometrica $68,1281-1292$.

[59] Rocheteau, Guillaume (2011). "Payments and liquidity and adverse selection," Journal of Monetary Economics, 58(3), 191-205.

[60] Roth, All (1995). "Bargaining experiments," Handbook of Experimental Economics, John Kagel and Alvin E. Roth, editors, Princeton University Press, 1995, 253-348.

[61] Rupert, Peter, Martin Schindler, Andrei Schevchenko, and Randall Wright (2000). "The search-theoretic approach to monetary economics: A primer," Economic Review of the Federal Reserve Bank of Cleveland, 4th Quarter, 10-28.

[62] Shao, Enchuan (2014). "The threat of counterfeiting in competitive search equilibrium," Journal of Economic Dynamics and Control 47, 168-185.

[63] Shi, Shouyong (1995). "Money and Prices: A Model of Search and Bargaining," Journal of Economic Theory $67,467-496$.

[64] Shi, Shouyong (1997). "A divisible search model of fiat money," Econometrica 65, 75-102.

[65] Trejos, Alberto (1997). "Incentives to produce quality and the liquidity of money," Economic Theory $9,355-365$.

[66] Trejos, Alberto (1999). "Search, bargaining, money and prices under private information," International Economic Review 40, 679-695.

[67] Trejos, Alberto, and Randall Wright (1995). "Search, bargaining, money and prices," Journal of Political Economy 103, 118-141.

[68] Trejos, Alberto, and Randall Wright (2014). "Search-based models of money and finance: An integrated approach," Federal Reserve Bank of Minneapolis Research Department Working Paper 709.

[69] United States Treasury Department (2006). The use and counterfeiting of United States currency abroad, Part 3.

[70] Velde, François, Weber, Warren, and Randall Wright (1999). "A model of commodity money, with applications to Gresham's law and the debasement puzzle," Review of Economic Dynamics 2, 291-323.

[71] Williamson, Stephen and Randall Wright (1994). "Barter and monetary exchange under private information," American Economic Review 84, 104-123. 